Extrême-Orient Extrême-Occident

\section{Extrême-Orient Extrême-Occident}

39 | 2015

Corps souffrants dans les littératures de la Chine et du Japon au XXe siècle

\title{
Le corps souffrant chez Lu Xun : allégorie muette de l'obstacle et appropriation de la modernité
}

The Suffering Body by Lu Xun as a Silent Allegory of Constraint and Appropriation of Modernity

宇樂文「魯迅的作品與受苦的身體：現代性的適應與阻礙的無聲寓 言」

Victor Vuilleumier

\section{(2) OpenEdition}

\section{Journals}

Édition électronique

URL : http://journals.openedition.org/extremeorient/512

DOI : 10.4000/extremeorient.512

ISBN : 978-2-84-292-449-2

ISSN : 2108-7105

Éditeur

Presses universitaires de Vincennes

Édition imprimée

Date de publication : 1 octobre 2015

Pagination : 47-84

ISBN : $978--84292-447-8$

ISSN : 0754-5010

Référence électronique

Victor Vuilleumier, « Le corps souffrant chez Lu Xun : allégorie muette de l'obstacle et appropriation de la modernité », Extrême-Orient Extrême-Occident [En ligne], 39 | 2015, mis en ligne le 01 octobre 2017, consulté le 04 mai 2019. URL : http://journals.openedition.org/extremeorient/512 ; DOI : 10.4000/ extremeorient.512 


\title{
Le corps souffrant chez Lu Xun : allégorie muette de l'obstacle et appropriation de la modernité ${ }^{1}$
}

\author{
Victor Vuilleumier
}

\section{Émergence d'un nouveau discours du corps en Chine}

Le corps de la «modernité mondialisée » (global modernity $)^{2}$ fait en Chine l'objet d'un développement croissant suite à la défaite chinoise devant le Japon en 1895 . C'est à ce moment que se mettent en place des représentations du corps comme élément constitutif d'une modernité devenue ensuite normative dans la Chine républicaine (1911-1949) : c'est « l'esthétique du corps» sublime (tipo meixue, aesthetics of corporeality) ${ }^{3}$, comme exaltation de la puissance, de la vitalité et de la force physique ${ }^{4}$. Cette esthétique se matérialise dans les discours et politiques du « renforcement » et de la «militarisation » qui se succèdent en Chine, des années 1860 à la fin des années $1910^{5}$, dans un contexte de valorisation progressive des armes et des valeurs martiales, aussi bien chez les réformistes que les révolutionnaires anti-mandchous, ou dans les mouvements du militarisme ou de la Nouvelle culture de la fin des Qing (16441911) et de la République. Jusqu'alors, de telles valeurs étaient tenues comme inférieures aux vertus lettrées. La représentation dominante est celle du corps fort, que l'individu est appelé à se donner pour constituer un corps national

1. L'auteur remercie vivement Cécile Sakai, Gérard Siary et les deux relecteurs anonymes pour leurs précieux retours.

2. Fran et Heinrich 2006 : 10. Voir Dirlik 2010, qui considère, contre la théorie des « modernités plurielles », que toute revendication de modernité, malgré ses diverses adaptations hybrides locales et nationales, témoigne de l'universalité d'un même processus dû à la mondialisation des rapports.

3. Wang $1997: 55-59$; Wang $2003: 376$; Wang $2004: 279$.

4. Tel l'appel de Yan Fu à renforcer la « force du peuple» (minli 民力), dans « Aux origines de la puissance » (Yuan qiang, 1895).

5. Sur les liens entre modernisation et militarisation, voir Lou et Knechtges 2012 : 57981. 
puissant, assurant à la Chine une survie dans la lutte entre sociétés et nations vues comme des organismes.

Cette esthétique du corps sublime, horizon du discours chinois moderne sur le corps, se forge en opposition à celle du corps évanescent qui, selon la sensibilité cultivée prémoderne, est perçu comme faible, malade, déformé, et honteux. Il devient le signe du "malade de l'Asie », que métaphorise la figure de la femme aux pieds bandés ou du fumeur d'opium. Se met en place une rhétorique du corps, selon une polarité fort/faible, masculin/féminin, occidental/chinois. Ce corps de genre féminin devient une métaphore, un objet du discours et de l'action politique. Cependant, le corps fort individuel, bien que valorisé, est réduit au rôle de métaphore de la volonté et de "l'âme » $(h u n)^{6}$ nationale. Il est soumis à la discipline de la modernité, «nationalisé » 7 et en définitive, inféodé à la "force spirituelle» (xinli). Cette importance du corps et de l'esprit dans la discussion de questions morales et culturelles est manifeste également chez les auteurs et artistes chinois dès les années 1910, constituant une revendication des valeurs de modernité et d'émancipation ${ }^{8}$. La médecine occidentale, mais aussi la représentation anatomique en peinture ou la reconnaissance des « instincts » (benneng) par la Nouvelle culture, en sont l'exemple.

\section{Configuration textuelle du corps chez Lu Xun}

La littérature importe des représentations de champs hétérogènes, dont elle se démarque ou non. Ceci dit, il n'y a pas de norme sociale qui fixe une fois pour toutes l'écart ou la conformité d'une représentation littéraire? Par sa clôture sémiologique, le texte, aussi dépendant du contexte soit-il, crée un "imaginaire littéraire ${ }^{10}$ » original. L'œuvre à son tour invente des thèmes (réseau d'images, de perceptions, en rapport aux éléments, au temps, à l'espace, au corps) insérés dans des mythologies propres. Étudier le sens et la représentation du corps dans une œuvre singulière consiste à définir un style, un tropisme, en parallèle également avec les représentations littéraires et extralittéraires. Ce style du corps ouvre au travail d'interprétation de sa signification globale dans l'œuvre. Le cercle herméneutique se complique encore de par

\footnotetext{
6. Vuilleumier 2011b : 271-275.

7. Huang 2006.

8. Heinrich 2002 : 167 ; Vuilleumier $2010: 131-7$.

9. Sur « l'écart » entre représentations littéraires et sociales, voir Chartier 2009: 326.

10. Ricœur $1998: 158$.
} 
la «modernisation hybride ${ }^{11}{ }$, en l'occurrence, celle des représentations du corps et de la littérature chinoise moderne. Et si le texte est ce par quoi un lecteur s'invente lui-même, on peut décrire l'horizon d'attente et de réception par le « trajet intentionnel ${ }^{12}$ » des textes, et par exemple, leur « effet de texte ${ }^{13}$ » sur le lecteur. Mais il faut aussi sonder les «intentions de l'œuvre ${ }^{14}$ » ses « schèmes » qui débordent les desseins explicites de l'auteur et son contexte.

Les textes de Lu Xun (1881-1936), l'un des «fondateurs » de la Nouvelle littérature chinoise (Xin wenxue), se prêtent à une telle enquête sur le corps littéraire souffrant. Mais ils ne témoignent pas d'une écriture du corps, au sens d'une narration corporelle qui prend le corps comme objet principal de son récit ${ }^{15}$, aborde des sujets tabous ou libère le corps et ses énergies à la façon d'un Antonin Artaud. Il y a trois raisons pour cela. D'abord, à peu d'exceptions près, aucun récit ne s'inscrit dans une phénoménologie de la corporalité qui exprime la souffrance en termes explicites. Le « corps souffrant » y est essentiellement métaphore et allégorie, c'est-à-dire signe lisible d'autre chose de lui-même - allégorie -, ainsi que réalité culturelle multiple - métaphore - avant d'être biologique : autant d'images corporelles de la souffrance. «Souffrance » doit s'entendre donc comme signification indirecte de la situation d'aliénation des Chinois, issue du contexte socio-historique, du « caractère national » malade, du silence, lourd et pesant, de l'insoutenable obstacle du corps. Ces souffrances sont portées et désignées par des métaphores et allégories corporelles.Le « corps romanesque $^{16}$ » chez Lu Xun dessine ainsi une « anatomie de la douleur ${ }^{17}$ », cartographiable avec ses idiosyncrasies, son schéma, ses «postures », et ses thèmes corporels ${ }^{18}$, au premier chef la maladie et son corollaire, la guérison. De plus, la question du corps n'est pas séparable de celle de la quête d'une l'expression de la « voix du cœur» (xinsheng, xin étant le «cœur-esprit»), car la souffrance est aussi celle du silence. Enfin, il est d'autant plus difficile de

11. « La conception de modernisation hybride (hybride Modernisierung) semble appropriée pour rendre compréhensible ce processus dynamique d'interdépendance transculturelle et de croisement de ressources internes et externes qui a marqué le développement de la Chine au XXe siècle » (Heubel $2015: 4$ ).

12. Starobinski $1978: 24$.

13. Quignard $1997: 147$.

14. Rousset $1995: 16$.

15. Comme Champs de vie et de mort ou Samsara (Shengsichang, 1935) de Xiao Hong (1911-1942). Traduction anglaise : Xiao 1979.

16. Kempf $1968: 5-7$.

17. Assoun $2009: 49,128$.

18. Richard $2010: 11$. 
distinguer entre traumatisme individuel - ou « allégorie nationale ${ }^{19}$ » à portée collective - et corps de l'écriture que Lu Xun refuse de faire la part entre subjectivité séparée et destin collectif, comme si le corps particulier était à chaque fois la manifestation du corps social. Les intrications du corps national et du corps de l'écriture, présentes dans toute l'œuvre, n'imposent pourtant pas une lecture qui privilégierait l'allégorie nationale sans autre forme de procès. Au-delà de celle-ci, qu'elle examine à partir de la représentation du corps décapité et du corps divisé, la présente étude tient aussi à défendre une interprétation de Lu Xun qui en révèle la littérarité, à savoir la mise à mort symbolique du corps par la Lettre et la Loi.

\section{Le traumatisme moderne : à la recherche de la voix}

\section{Le corps décapité}

Le motif de la décapitation exprime le mieux le thème traumatisant du corps ayant perdu son intégrité. Il apparaît pour la première fois dans la « Préface » («Zixu ») à son premier recueil de nouvelles publié en août 1923, Appels (Nahan), dans cette scène censée se passer en janvier $1906^{20}$ :

Je nourrissais un beau rêve : une fois diplômé [il étudiait la médecine à Sendai, au Japon, où il était inscrit depuis 1904], je rentrerais pour guérir les souffrances (jiku) des malades abusés comme mon père [par la médecine traditionnelle], me faisant médecin militaire en temps de guerre, sans jamais cesser de renforcer la confiance de mes concitoyens dans la réforme [sur le modèle des réformes de l'ère Meiji au Japon, 1868-1912]. [...] à l'époque on utilisait des projections pour montrer l'aspect des micro-organismes [...] au moment de la Guerre russo-japonaise [1904-5], les images des hostilités étaient relativement nombreuses et, me trouvant dans cette salle de cours, je devais souvent partager la joie de mes camarades en applaudissant et en criant. Une fois, à ma surprise, je me retrouvai tout d'un coup face à face, sur l'image, avec nombre de ces Chinois qui me manquaient depuis longtemps : l'un d'eux était attaché, entouré de beaucoup d'autres, tous au physique robuste (qiangzhuangde tige) mais au regard apathique (ou esprit, mamude jingshen). D'après les explications, le prisonnier était un espion militaire au service des Russes, que les soldats japonais s'apprêtaient à décapiter et à exposer à la foule, et autour se trouvaient des gens venus savourer ce spectacle (haut fait, shengju).

19. Jameson $1986: 69$.

20. Pollard $2002: 30-1$. 
[...] je partis pour Tôkyô [en mars 1906], car à partir de ce jour-là, la médecine cessa de me paraître une affaire pressante : quoi qu'on fît pour renforcer leur corps et les rendre robustes, tous ces citoyens (ou nation, guomin) faibles et stupides (yuruo) ne pourraient servir que de matière première ou de contemplateurs $[\ldots]$ si nombreux qu'ils fussent à mourir de maladie, il n'y aurait là nul malheur. Notre première tâche était donc de changer leur esprit, et ce qui était le plus susceptible de provoquer ce changement était naturellement, à mes yeux d'alors, de promouvoir les belles-lettres, c'est pourquoi je voulus lancer un mouvement littéraire ${ }^{21}$

Dans ce passage, Lu Xun raconte sa conversion aux lettres, et son projet de lancer une revue littéraire baptisée Vita Nova (Xinsheng, en référence à Dante), en rejet de la médecine, c'est-à-dire de la science, pour une authentique modernisation. La même histoire est reprise plus tard dans « Professeur Fujino » (« Tengye xiansheng », 1926), avec des variantes qui lui permettent de préciser les raisons de son refus ${ }^{22}$. La médecine et la science, la modernité matérielle technique, constituent un projet aliénant à dépasser : or toutes s'occupent du corps. La science transmise à Lu Xun au Japon est inséparable d'un discours colonial et racialiste (le professeur Fujino mesure des crânes) : les Chinois sont perçus comme une masse indifférenciée, menaçante et déshumanisée de bactéries. Lu Xun, sujet de l'Empire mandchou, se devrait d'être superstitieux, de croire aux « esprits » (gui) et de refuser de disséquer. Fujino souhaite observer des pieds bandés, l'image par excellence de l'arriération renvoyée ainsi à Lu Xun ${ }^{23}$. Or, tous les intellectuels chinois réformistes depuis les années 1860 rejettent cette pratique, signe pour eux d'une tare collective. Dernier traumatisme, le rôle de Lu Xun se réduirait à celui de passeur d'une modernisation ne pouvant provenir que du Japon, dont il devrait rapporter en Chine la science.

Lu Xun se montre prisonnier d'une double contrainte (double bind). Il évoque son « rêve » de moderniser son pays par la médecine, en particulier sur le champ de bataille, ce qui est cohérent avec le discours du corps fort ; mais ce projet ne lui appartiendrait plus s'il devait n'être qu'un agent de l'action civilisatrice du Japon en Chine. Lu Xun, qui dénonce par ailleurs le chauvinisme chinois, n'en demeure pas moins attaché au rôle et au prestige culturels de la Chine. Lorsqu'il choisit un projet de renaissance par les lettres, il affirme la possibilité d'une modernité chinoise culturelle. Et même s'il entend faire prévaloir une modernisation culturelle et non scientifique, il met un point d'honneur à rappeler que certains des premiers manuels de

21. Tr. S. Veg (Lu 2010 : 14-15). Lu 2006e : 2-3. Les parenthèses et crochets sont de moi.

22. Lu 2007e : 79-86; Lu $1976: 145-148$.

23. Heinrich $2002: 184-190$. 
médecine introduits au Japon furent traduits du chinois ${ }^{24}$. Il veut renverser les rôles en accordant plus d'importance à l'esprit plutôt qu'au corps. Le rejet de la représentation du corps décapité, ainsi que de la culpabilité et de la honte qu'elle provoque chez Lu Xun, va de pair avec le rejet de la science et de la médecine. Lu Xun rejette ainsi et l'exhibition de la violence et l'intercession médicale ${ }^{25}$. Sa réaction ne s'explique pas que par son souci d'écrivain désireux de réaffirmer le rôle des lettres et promouvoir le « retour au classique » (fugu) face au medium moderne et «scientifique » de la diapositive qui incarne la technique visuelle moderne (visuality) ${ }^{26}$. Par sa force suggestive, cette dernière se fait le relais de la violence. En effet, l'image réaliste, qui reproduit le drame à chaque projection, fait du spectateur le complice de l'exécution. De plus, elle assigne Lu Xun à une position intenable : il se sent le membre désigné de ce pays présenté comme arriéré et faible, puis rabaissé au niveau de la foule spectatrice passive du supplice, et obligé d'applaudir. La gêne provient autant de la nature du spectacle visualisé que de la forme de la représentation.

\section{Du corps sublimé au corps divisé}

Le corps condamné à la décapitation constitue un mythe, une scène primitive, un manifeste intellectuel et esthétique. L'image du corps condense les deux causes du traumatisme : la violence et l'apathie supposée des Chinois, due aux défauts de leur «nation ». Elles expliquent d'ailleurs la faiblesse de la Chine face aux impérialismes. La figure du corps amputé de sa tête métaphorise la crise : la division et de la rupture du sens, la « perte des origines ${ }^{27}$ ». Wang Ban a démontré que Lu Xun passe d'une esthétique du sublime, fondée sur l'unité «symbolique » du sens (cœur) et du signe (corps), à une esthétique « allégorique », basée sur leur rupture définitive ${ }^{28}$. Représenter le corps divisé équivaut à rejeter « l'esthétique du corps » en tant qu'il est « signe du sublime ${ }^{29}$, ainsi que toute possibilité de conserver l'unité harmonieuse du corps et l'esprit, du signe et du sens, de l'individu et de l'universel.

24. Lu 2007e : 80 ; Lu $1976: 142$.

25. La question de la représentation et de ses modalités est au cœur des préoccupations littéraires de Lu Xun qui veut représenter le réel social et ses violences, sans en reproduire la violence et en être le complice (Anderson 1990).

26. Voir Chow $1995: 4-18$.

27. Wang $2004: 21$.

28. Wang $1997:$ 70-79. Il emprunte ces termes à Walter Benjamin.

29. Ibid. : 55-59. 
Lu Xun s'était tout d'abord fait l'écho de cette esthétique sublime dans ce qui est sans doute son premier texte, publié durant son séjour japonais (19031909) et rédigé en chinois classique, comme le seront ses textes jusqu'en $1918^{30}$. Sa nouvelle, «L'âme de Sparte » («Sibada zhi hun », 1903) ${ }^{31}$, adaptée des Histoires d'Hérodote (VII, 198-233), valorise l'esprit de sacrifice de Léonidas et ses hommes pour leur pays ${ }^{32}$. Ce texte paraît peu avant la guerre russo-japonaise (1904-1905), et transpose les acteurs présents dans le passé : les « Barbares » perses sont les Russes, qui menacent les « Grecs », à savoir les Chinois, appelés à défendre la Mandchourie, comme avant eux les Spartiates, les Thermopyles. Lu Xun reprend le discours nationaliste répandu parmi les étudiants et les intellectuels chinois au Japon, à l'origine du « Mouvement du militarisme » (Junguomin yundong, 1902-1919) ${ }^{33}$. Mais Lu Xun prend ses distances avec l'esprit martial et l'exaltation de la force physique, dénonçant le militarisme dans « De la dénonciation des voix malignes » (« Po e'sheng lun $», 1908)^{34}$, ce qui n'interdit pas un souci nationaliste. Il fait de même avec la science, pour en venir à l'affirmation progressive de la culture, dans « Des déséquilibres dans la culture » («Wenhua pianzhi lun», 1908) et « La puissance poétique de Mara » («Moluo shili shuo», 1908) ${ }^{35}$.

\section{Le corps obstacle à la voix et à la vie}

La question de l'expression du cœur par le corps est centrale chez Lu Xun car il s'agit pour lui de résoudre le conflit entre baihua (« chinois moderne ») et wenyan («langue classique »), entre parole et silence. Dans «Épitaphe »

30. À l'exception de son journal, de poèmes, de ses écrits officiels, académiques ou pédagogiques, qu'il rédigera dans cette langue toute sa vie.

31. Lu 2007d: 7-14.

32. Lu 2007d: 7-8.

33. Sur ce mouvement, son esthétique et sa mise en application dans l'éducation, voir Huang 2006 : 45-51. L'Armée révolutionnaire (Gemingjun) de Zou Rong (鄒容, 18851905), paru un mois plus tôt à Shanghai, donne le ton. Le mouvement, soutenu par la modernisation militaire en Chine depuis les années 1860, par le nombre croissant des troupes au cours du XIX $X^{\mathrm{e}}$ siècle, et surtout par la revalorisation des armes à l'issue de la Nouvelle politique (Xinzheng, 1901) des Qing, est préconisé par des intellectuels et militaires chinois, appelant à prendre exemple sur le Japon, tel Liang Qichao 梁啟超 auteur d'une « Brève histoire de Sparte » («Sibada xiao zhi », 1902).

34. Lu 2011 ; Lu 2006b : 23-38.

35. Lu 2006d : 43-118; Lu $1981: 61-143$. Nous proposons cette traduction du titre en français de $l i$ («force ») par «puissance», pour relever la connotation sublime que ce mot revêt dans le texte de Lu Xun, et de «Moluo shi » par « poésie de Mara », plutôt que « des poètes de Mara », shi 詩 étant la «poésie » et non le «poète ». 
(«Mujiewen », 1925), poème en prose en baihua,le narrateur rêve qu'il arrive devant une tombe, dont la stèle inscrite sur ses deux faces porte en wenyan l'histoire du mort enterré. Ce dernier, mort de son propre « venin » $(d u)$, est devenu une «âme errante » (youhun). Il a ensuite « dévoré » (zi shi) son propre « cœur» (xin) se plaçant dans une situation intenable :

[...] s'arracher le cœur pour le manger, afin de découvrir sa propre saveur (benwei). Mais si la souffrance est trop vive, comment [le] pourrait-on [...]

[...] la souffrance une fois calmée, le manger lentement. Mais [s'il] a ranci entretemps, comment [la connaître ${ }^{36}$ ?

Ce paradoxe définit le problème de l'expression ${ }^{37}$ : le cœur ou la signification ne peuvent se dire sur le vif, comme le «feu mort » immobilisé mais toujours ardent sous la glace ${ }^{38}$. Ceci est signifié au rêveur, à la fin poursuivi, terrorisé, par le mort à la poitrine ouverte sans « cœur ni foie » (zhong wu xin gan) - c'està-dire dénué de «sincérité » (xin'gan) au sens figuré. L'écriture classique est réduite à une ruine lacunaire : telle la poitrine vide et défoncée, l'épitaphe est morcelée. Le mort répond à la question sans « remuer les lèvres » (kouchun bu dong). Ce mort-vivant est le passé qui hante le rêveur. Il est le fantôme du signe chinois et de sa culture, revenu poursuivre le narrateur moderne cherchant à écrire en baihua. Le narrateur pourtant ne parvient à se détacher du wenyan : après lecture des deux faces de la stèle, parole du mort, il introduit du wenyan dans sa propre voix ${ }^{39}$ (rythme tétrasyllabique archaïsant), ironie de Lu Xun, mais signe de fascination morbide. Dans «Écrit après La Tombe " «Xie zai Fen houmian », 1926) ${ }^{40}$, Lu Xun dit craindre « d'empoisonner » (duhai) la jeunesse du fait de son éducation classique imprégnant ses écrits ; les écrivains à venir, pour faire entendre une « voix nouvelle » (xin sheng) devraient «faire de la parole des vivants leur source » (huorende chunshe zuowei yuanquan), pour s'approcher de la « langue (parlée) » (yuyan) et donc de la vie (shengqi $)^{41}$. Au regard de «La puissance poétique de Mara », l'homophonie «vie» (sheng) et « cri » (sheng, « voix ») prend tout son sens : c'est la parole vive, qu'il faut trouver comment exprimer par une nouvelle langue écrite, ce que devrait

36. Tr. Leys (Lu 1975). Voir Lu 2006c : 45.

37. Davies $2013: 273$.

38. Voir « Le feu mort » («Sihuo », 1925), incarnant « l'esprit de lutte » pour Qian 2004 : 283-284.

39. Lee $1987: 93$.

40. Il s'agit de la postface au recueil de Lu Xun intitulé La Tombe (Fen 墳).

41. Lu 2006d : 299-300. 
être le baihua. «Épitaphe » est l'allégorie, véritable emblème, de la défense et illustration du baihua. Le corpus classique, le wenyan, voire même les caractères chinois, sont métaphorisés par un corps vide d'esprit, un revenant, icône de l'effroi ressenti face au texte du passé.

Lu Xun évoque sa difficulté à écrire, son impression de «vide» (kongxu) éprouvée au moment «d'ouvrir la bouche » $\left(\mathrm{kai} \mathrm{kou}^{42}\right.$, ce que le corps mort ne peut faire. Ce vide peut s'expliquer par un scrupule ou une inquiétude ${ }^{43}$, mais aussi par un sentiment de déperdition causée par l'expression. L'écriture de Lu Xun s'adresse aux «fantômes, monstres, hiboux et serpents ${ }^{44}$ - êtres marginaux, souvenirs du passé. Cet imaginaire « gothique » signale la dimension sépulcrale de l'écriture (« Épitaphe »), qui enterre et donne pour mort ce qui est dit. Or, dire et écrire relève de la vie : d'où les images de la croissance végétale et l'expression des sentiments, dans "Prologue à $L a$ Mauvaise herbe » («Tici», 1927) ${ }^{45}$. La vie du texte se nourrit de la «chair et du sang » des «morts » enterrés (chensiren de xue he rou $)^{46}$, c'est-à-dire du passé, des attentes et espoirs, mais aussi de la «vie et de la mort », de « la lumière et de l'obscurité ». Lu Xun recourt au paradoxe pour parler de l'écriture, espérant à la fois son apparition et sa disparition, pour dessiner un espace intermédiaire improbable, une fine pointe de l'expression menacée de disparition. Écrire est une activité biologique, née de la mort de l'ancien, de sa décomposition, et soumise elle-même à la corruption : ce processus est nécessaire pour permettre l'expression fidèle et sincère de "la chair et du sang » « mis à nu ${ }^{47}$. En rappelant ce qui a vécu, le texte signale sa propre mort. De cela, le corps, matière abandonnée, est la métaphore inquiète. Dans le texte même d' « Écrit après La Tombe », Lu Xun érige une stèle ${ }^{48}$, sorte de bilan rétrospectif de ses textes classiques et modernes réunis dans le recueil. C'est son tombeau, par lui composé, qui recèle le «corps » (quke) réduit en «poussière » de ses souvenirs, la «marque » de sa vie passée. Le mot yuhen, qu'il emploie, comprend «hen», la cicatrice, la marque laissée ${ }^{49}$, « la mémoire

42. Lu 2006c : 1 ; Lu $1973: 12$.

43. Lu 2006d : 299.

44. Lu 2006d : 298.

45. Lu 2006c : 1-2.

46. Lu 2006c : 1 .

47. Lu 2006d : 298.

48. La postface s'achève sur la citation d'un poème de Lu Ji (261-303), faisant l'éloge posthume de Cao Cao 曹操 (187-226) ayant ordonné de simplifier les rituels funèbres à sa mort, à qui Lu Xun s'identifie ici !

49. Lu 2006d : 301. 
de la perte p $^{50}$ » de ses aspirations à faire naître une « voix du cœur » par le « retour à l'antique ». Lu Xun enterre l'écriture classique.

Le corps mort du passé est un obstacle à la voix et à la vie. Dans «Chine muette » («Wushengde Zhongguo », 1927), Lu Xun se demande comment faire entendre aux Chinois leur « propre voix » au présent. Ils doivent se libérer aussi bien de leur propre corpus classique, que de la parole colonisée, celle que les Occidentaux tiennent sur les Chinois comme de celle que les Mandchous ont effacée par les «procès littéraires » (wenziyu) ou la pratique des caractères tabous de l'ancien régime : supprimer ou interdire un caractère accompagne le supplice des corps ${ }^{51}$. De même que dans «La puissance poétique de Mara », l'auteur appelait à la « voix du cœur », il compare le fait de donner une voix à la Chine muette à demander à un « mort » de « renaître » (huoguolai $)^{52}$. Pour lui, proche de Zhuangzi sur ce point ${ }^{53}$, les textes anciens sont les « paroles des morts antiques » (gudaide sirende hua), et les « textes en baihua» (baihuade wenzhang) celles des «vivants » (xiandaide huorende hua $)^{54}$.

Dans cette conférence, Lu Xun appelle la jeunesse à rejeter les « lettres classiques » $(\text { guwen })^{55}$ afin de faire entendre sa voix pour « vivre ». La quête de l'expression marque ce désir de vie et de renaissance de l'esprit et du cœur, qui permet à la culture de renaître en retour. « La puissance poétique de Mara » est introduite par cette citation modifiée du Zarathoustra : «Ceux dont les recherches ont épuisé les antiques origines vont sonder les sources futures pour de nouvelles origines. Mes frères, sous peu une nouvelle vie apparaîtra et de nouvelles sources surgiront des profondeurs ${ }^{56}$. » Lu Xun modifie l'original ${ }^{57}$, remplaçant « les nouveaux peuples » (nеuе Völker) par « l'apparition d'une nouvelle vie » (xinsheng zhi zuo). La nouvelle vie doit être la nouvelle voix. Or, ce thème de la renaissance de l'esprit et de la voix ${ }^{58}$ est exprimé par le motif

50. Agamben $2015: 9$.

51. Lu 2007b : 171-2

52. Lu $1973: 7$. Voir Davies $2013: 262$.

53. Zhuangzi 1998, t. $2: 357$.

54. Ibid.

55. Faut-il rejeter les caractères ou simplement le chinois classique ? Comme le dit Lu Xun, pour pouvoir ouvrir une fenêtre, il faut menacer de supprimer le toit : d'où la traduction « lettres classiques », qui englobe le signe autant que les textes classiques.

56. Lu 2006d : 63. Voir Nietzsche $1999: 262$.

57. Von Kowallis $2013: 429$.

58. Quignard $1997: 82$. 
de la renaissance grotesque du corps ${ }^{59}$, parodique, figurant un mouvement perverti car mort.

Le mouvement est essentiel. Dans «Le passant» («Guoke », 1925), un homme anonyme avance d'est en ouest, suivant le trajet du soleil, en direction des «tombes ». Il est poussé par une « voix » qui l'appelle comme un daimôn. Semblable au fantôme errant d' « Épitaphe », il aimerait boire du « sang », mais se contente d'eau. Il ne cesse de marcher malgré ses pieds abîmés, symboliquement comme les pieds bandés du passé (une fillette lui tend un tissu pour les panser). L'image du marcheur aux pieds abîmés qui ne doit pas s'arrêter s'oppose aux motifs de l'immobilité et de la paralysie corporelles. Cependant, le corps en mouvement, mort ou vivant, est signe d'une situation impossible. Le corps du revenant est un corps qui ne peut s'exprimer («Épitaphe ») ; le corps rigide et muet est habité d'une tension mutique ("Tremblement au bord de l'abîme », « Tuibaixian de zhandong », $1925)^{60}$. Dans «Après la mort », le narrateur fait l'expérience de rester conscient après la mort, paralysé mais sensible. Ces motifs chez Lu Xun composent une série d'oppositions thématiques (vitalité/léthargie, flux/ mort, vie/nécrose, parole/silence), qui recoupent fondamentalement celle du corps mobile ou immobile ${ }^{61}$, plus encore, celle du corps qui peut entendre, ou faire entendre, une voix : le véritable mouvement est l'expression de la parole vive. Le style de la représentation du corps chez Lu Xun s'organise autour du mouvement de la parole, de l'expression (biaoxian) ${ }^{62}$. C'est une tension exprimée par des paradoxes et images contraires. La tension construit le thème de la lutte et de la révolte ${ }^{63}$. La figure du lanceur de " javelot » monté « au front du néant» (wuwu zhi zhen) dans « Un combattant comme ça » («Zheiyangde zhanshi », 1925) signifie ce mouvement d'affrontement corporel toujours renouvelé ${ }^{64}$. Le «vide» qu'affronte le narrateur est celui des faux-semblants des concepts moraux destinés à tromper les esprits. Mais ces concepts sont aussi des mots, renouvelés en permanence au fur et à mesure de leur destruction par le javelot évolutif et critique du combattant. Ils incarnent l'absence de sens et de voix sincère : ce « vide » à affronter est de même nature

59. Sur le même motif, voir« Après la mort » (« Sihou », 1925, Lu 2006c : 52-56) et «Résurrection » («Qisi », 1936, Lu 2007c : 133-145).

60. Davies $2013: 256$.

61. Gernet $1995: 271$.

62. Le souci de l'expression et de la représentation explique la dimension « visuelle » de nombreux textes de Lu Xun, comme pour dépasser l'immobilisme supposé de l'écrit chinois.

63. Sun $2007: 32$.

64. Étrangement proche de la tension sublime (voir Quignard 1997 : 58). 
que le revenant d' 'Épitaphe », c'est celui du corps et du signe. Le corps est métaphore du discours du Père, du passé, du wenyan, voire des caractères qui hantent le vivant et font obstacle à la « voix nouvelle», le baihua. Si l'on suit Simon Leys, qui voit chez Lu Xun une interrogation sur « l'âme » et la mort ${ }^{65}$, il apparaît que le corps en propre est visé. Il apporte l'aliénation et la mort. Véhicule de l'expression, il est aphasique, épitaphe illisible. Le corps luxunien souffre d'être sèma et sôma, il a perdu sa faculté à produire un "son » qui signifie ${ }^{66}$.

Cette expression frustrée définit le kumen (« dépression, mélancolie, angoisse $»)$, souffrance de $«$ l'esprit ${ }^{67}$. Ce terme est introduit parKuriyagawa Hakuson (1880-1923) dans Le Symbole de la mélancolie (Kumon no shôchô, 1921), que Lu Xun traduit et publie en 1924 (Kumende xiangzheng) ${ }^{68}$. Selon cette dernière version, toute création artistique est «l'expression » symbolique $\mathrm{du}$ sentiment de « kumen $»\left(\right.$ kumende biaoxian) ${ }^{69}$, « existentiel (shengde kumen $)^{70}$, apparu au contact des obstacles que la « force vitale » (shengmingli) rencontre ${ }^{71}$. Cette conception d'un élan vital frustré dans son mouvement rejoint l'idée que Lu Xun a développée ailleurs de l'existence comme procès d'évolution au cours duquel la vie prime sur toute chose. Aussi le refus du changement moral est-il selon Lu Xun contraire aux lois de l'évolution ${ }^{72}$. D'où l'intrication, voire la dialectique de la vie et de la mort (« Prologue à $L a$

65. Lu $2006: 151 ;$ Lu 1975 : introduction.

66. Quignard $1997: 23-4$.

67. Lu $2008: 234$.

68. L'ouvrage est traduit une première fois en 1921 (McDougall 1971 : 108). Lu Xun joue un rôle important dans la diffusion de ce concept en Chine, central pour la Nouvelle littérature des années 1920.

69. Lu $2008: 238$.

70. Lu $2008: 225$.

71. Kuriyagawa élabore la conception d'une force frustrée à l'œuvre dans toute production artistique et littéraire à partir de la théorie freudienne de la création comme sublimation et du trauma comme refoulement. Il trouve la notion de libido freudienne trop limitée : la «force vitale » intègre «l'élan vital » de Bergson. Le kumen est l'expression de la résistance des forces vitales à l'obstacle, comme la fièvre est la manifestation de la résistance de l'organisme à la maladie ( $\mathrm{Lu} 2008$ : 244). L'art est le symbole et la sublimation de cette force vitale et créatrice frustrée. Elle se comprend comme souffrance existentielle, et concrètement, comme lutte entre le désir d'expression et d'affirmation créatrice de l'individu, et la société. De même, la vie dans une société mécanisée produit l'aliénation du travail.Le kumen exprime les conflits et antagonismes de l'âme contre le corps, de l'individu contre la société, de l'esprit contre la matière.

72. Voir «Quels pères devons-nous être aujourd'hui » («Women xianzai zenyang zuo fuqin », 1919), Lu 2006d : 138. 
Mauvaise herbe »), de l'amour et de la mort («Vengeance»), qui constitue le mouvement même de la vie. Ainsi, «l'évolutionnisme » de Lu Xun ne fait-il qu'un avec l'éthique du « développement » de la «vie » (shengming) ${ }^{73}$. Puisque le corps en tant que signe écrit ancré dans le passé fait obstacle à l'expression renouvelée de l'actuel, il doit s'autodétruire s'il veut se faire expression du monde qui change. Et Lu Xun n'hésite pas à le soumettre à la plus autophage des représentations.

\section{Le refus du corps : pour une autre modernité}

Le motif du corps décapité exprime une crise intellectuelle, l'abandon de la médecine et de l'esthétique du corps. Il implique également une opposition entre corps et esprit, pour exprimer celle séparant « science » et « lettres ». Dès ses premiers textes publiés durant son séjour japonais, Lu Xun recourt à de telles métaphores qui donnent de l'importance à la culture, à la voix poétique, au « cœur-esprit » (xin), et ce à l'encontre du corps et de la civilisation matérielle. Il oppose au corps désigné comme une enveloppe uniquement matérielle, un champ lexical de « l'esprit » (jingshen) ou du « cœur» bien plus développé et positif ${ }^{74}$. Pour Lu Xun, le scientisme développe une conception matérialiste du corps inerte qui menace l'esprit de disparition. C'est le cas de ces lettrés réformistes qui, «bien que leur corps (quke) subsiste, ont perdu leur intelligence (lingjue) », et fort de leurs faibles connaissances en «physiologie » (shengli), vont répétant : « Le corps humain (renti) étant composé de cellules, où trouverait-on une âme (linghun) ${ }^{75}$ ? » Le corps c'est la civilisation du « matérialisme » (wuzhi) importée par erreur en Chine par ces réformistes, dont $\mathrm{Lu}$ Xun dénonce l'ignorance du réel intérêt qu'offre l'Occident. C'est aussi la « démocratie » (zhongzhi), la dictature des masses et de la « majorité » (duoshu), qui signera la ruine de «l'individualité » (gexing), selon le portrait que dresse de l'Occident du XIX ${ }^{\mathrm{e}}$ siècle un Lu Xun anti-moderne. Ce système politique et civilisationnel, véritable « déviation culturelle ( wenhua pianzhi), détruit la créativité ( « talent naturel », tiancai) de l'individu et toute possibilité pour lui de se nourrir de la bénéfique « influence des anciens rois » (xianwang zhi ze $)^{76}$. La Chine est donc malade, atteinte de «paralysie», du fait que le

73. Lu 2006d : 133. Voir Cao $2006: 267-268$.

74. Gao $2007: 2$.

75. Lu 2006b : 28 .

76. Cette influence est positive : le culte supposé de la matérialité a détruit la créativité en même temps que la bénéfique influence des anciens. La créativité de l'individu, 
développement de «l'esprit » est contrecarré par la matière : la «puissance » collective ne peut s'obtenir que par «l'établissement de l'homme » (liren) ${ }^{77}$. Le développement de l'individu inspiré par le poète « Mara» est pour Lu Xun l'unique solution à la crise nationale. La « voix [de son] cœur », la force sublime et virile de son esprit, transmise par sa parole poétique, se fera entendre de ses concitoyens, leur transmettant sa vertu salvatrice, revigorant ainsi leur esprit et leur corps paralysés. Le «combattant du règne de l'esprit » (jingshenjie zhi zhanshi) est appelé à remplacer le réformisme des institutions, de l'industrie et du commerce, et le militarisme :

Où débusquer en Chine le combattant du règne de l'esprit ? Qui nous fera entendre le timbre (sheng) de la plus parfaite sincérité (cheng), pour que nous accédions revigorés au bon et au bien (shanmei gangjian) ? qui produira une voix (sheng) assez fervente pour nous extirper de notre froide dévastation? Sur la terre de nos pères (jiaguo) devenue vaine (huang), il n'est pas encore, le Jérémie qui déclamera l'ultime lamentation, pour léguer aux générations sa dénonciation du monde (tianxia). Soit il n'est pas né ; ou s'il l'est, la tourbe l'aura fait taire. Dans les deux cas, la léthargie de la Chine s'ensuit. Comme l'on n'a su que concentrer laborieusement nos soins sur notre enveloppe corporelle (quke) [depuis les modernisations ayant suivi les guerres de l'opium puis avec les réformes de 1898 et 1901], l'esprit (jingshen) de jour en jour régresse, au point de ne pouvoir se maintenir contre les nouveaux courants. La multitude parle de « réforme » (weixin) : mais ce n'est que l'expression de paroles (sheng) de qui confesse ses péchés commis depuis toujours, comme s'il voulait se réformer (gaihui) $^{78}$. Et pourtant avec ces réformes, tous nos espoirs avaient commencé : nous attendions des savants (shiren) qu'ils nous présentassent une nouvelle culture. Cependant l'examen de ce qu'ils ont rapporté depuis plus de dix ans que dure cette introduction continue, ne révèle rien d'autre que [des

pour laquelle $\mathrm{Lu}$ Xun préconise des nourritures spirituelles occidentales, a besoin également de la « rosée » antique pour (re)naître. Plus haut dans le texte (ibid. : 55), est défendue l'idée d'établir une « nouvelle école » en «s'appropriant le moderne et revenant à l'antique » (qu jin $f u g u$ ), soit une renaissance culturelle via la redécouverte des ressources classiques. "Li ren » est à lui seul une expression et un concept « orthodoxe» (Gao $2007: 3)$. Cette volonté initiale de renaissance explique pourquoi Lu Xun est lu comme source de réflexion pour une modernité autre.

77. Lu 2006d : 56.

78. Mes italiques pour relever la critique du ressentiment moral dissimulé derrière le projet de réforme. 
ouvrages traduits en chinois par les anciens étudiants revenant du Japon, traitant $\left.{ }^{79}\right]$ de recettes de gâteaux et de surveillance pénitentiaire ${ }^{80}$.

Cette transmission d'une esthétique de la « sincérité » du poète à l'auditeur, en vue de la transformation éthique de ce dernier par la voix poétique, reprend l'idée de la «poésie comme expression du sentiment » ou de la «volonté » (shi yan zhi $)^{81}$. Lu Xun défend un projet moderne lié à celui de Zhang Taiyan (18681936), de redéfinition des «études nationales » (guoxue), de « renaissance » culturelle, de " retour à l'antique », dont le style de prose classique dit Wei Jin (dirigé contre la prose parallèle Tongcheng « conservatrice ») ${ }^{82}$ est l'expression littéraire. L'originalité de Lu Xun cependant est de vouloir établir une «nouvelle école » (xinzong), qui intègre la tradition étrangère de parole romantique inspirée et épique de la «poésie Mara ». Le « Spartiate » est devenu poète romantique, « homme » (ren) confucéen et «surhomme » (chaoren) nietzschéen. La jonction entre nationalisme et individualisme se fait par le biais de la figure du héros culturel et non plus martial. Lu Xun ne rejette pas la science en tant que telle ${ }^{83}$, mais la modernisation matérielle qui se voudrait uniquement technique et physique. Il change de paradigme, de l'étude de la science à celle de «l'esprit » ${ }^{84}$. Il établit une critique culturelle des « voix malignes » des réformistes chinois et des « travers culturels » modernes, et s'inspire du volontarisme, de l'individualisme, de l'existentialisme et de l'anti-rationalisme occidentaux (Nietzsche, Ibsen, Stirner, Kierkegaard, Schopenhauer $)^{85}$. Lu Xun partage avec les penseurs réformistes des années 1890-1900 un même rejet de la matière au nom de «l'esprit» ou de la «force spirituelle »- mais lui, poétise la « force ${ }^{86}$. Lu Xun a sublimé la puissance physique en force poétique du cœur, et dans ses textes à partir de 1918, la

79. Lu 2006d : 118 .

80. Lu 2006d : 100-101.

81. Yuan $1996: 17-24$.

82. Chen $1998: 291-292$.

83. À son retour en Chine en 1909, Lu Xun enseigne la physiologie pendant quelques temps et rédige un polycopié en wenyan, le Manuel illustré de physiologie humaine (Ren sheng xiangxiao, 1909), Lu 2006a : 75-225.

84. Sur le passage de kexue à xinxue, voir Gao 2007 : 1-4.

85. Wang 2008 : 53-111. Le Japon est d'ailleurs le médiateur par lequel il découvre ces références via des versions allemandes ou japonaises, et qui lui transmet certaines thèses sur le développement de la culture, comme Takayama Chogyû (Pollard 2002 : $35)$.

86. L'affirmation du corps fort est avant tout celle de l'âme nationale. Ces auteurs témoignent d'un puritanisme et d'un rejet du corps individuel certains. 
médecine devient une métaphore : le vrai médecin est l'écrivain soignant l'esprit, et non le praticien traitant les corps. La métaphorisation est un moyen d'appropriation du discours de la modernité scientifique.

\section{Remède à la maladie : métaphoriser la science dans le « Journal d'un fou »}

Le choix de la langue dans laquelle écrire accompagne ce changement esthétique de Lu Xun. Jusqu'alors, le wenyan incarnait son projet de renaissance porté par la figure du poète surhumain, héraut de sa nation menacée. Avec le Mouvement pour la Nouvelle culture (Xin wenhua yundong) et de la Nouvelle littérature du 4 Mai (Wusi), Lu Xun s'astreint au baihua ${ }^{87}$, qu'il contribue à développer, pour former une nouvelle langue littéraire dans le contexte de l'établissement d'une koinè moderne, la « langue nationale » (guoyu), définie dans les années suivant la fondation de la République. À partir de 1918, le chinois classique ou littéraire dans les textes publiés de Lu Xun cède la place au baihua, devenant l'incarnation de nouvelles valeurs à défendre, et le classique, la métaphore de celles devant être rejetées.

C'est le cas dans le « Journal d'un fou » (« Kuangren riji », 1918) ${ }^{88}$, premier texte littéraire en baihua de Lu Xun et l'un des principaux hypotextes chinois $\mathrm{du} \mathrm{XX}^{\mathrm{e}}$ siècle. La nouvelle oppose l'éveil de l'individu au « cannibalisme » inculqué à tous par les « rites » $(\text { lijiao })^{89}$ : d'un côté, la prise de conscience inédite de l'individu $^{90}$, qui espère interrompre ce processus en rétablissant la linéarité de l'histoire par l'écriture ; de l'autre, une histoire immobile et cyclique $^{91}$, une éducation et un système familial, économique et social, qui amène chacun à dévorer son semblable. Cependant, l'individu, diariste de la nouvelle qui connaît un tel éveil et que présume « fou » l'éditeur fictif du manuscrit retrouvé, n'est plus un surhomme ${ }^{92}$ : il se reconnaît complice et responsable. Il a pris conscience de la faute collective, mais aussi de sa propre culpabilité, en même temps qu'il se sent menacé par les autres, établissant un rapport complexe de l'individu à la société.

87. Admussen 2009.

88. Lu 2006e : 9-21; Lu $2010: 19-31$.

89. L'association explicite du cannibalisme aux « rites » du confucianisme est le fait du premier article critique publié sur la nouvelle (Wu 1982). La critique féroce dirigée contre les rituels est une caractéristique des lettrés excentriques de la période Wei Jin.

90. Veg $2010: 93$.

91. Zhang $1992: 133$.

92. Zhang $2001:$ : 78-79. 
L'opposition des valeurs s'exprime par ces motifs : d'un côté, des figures de l'autorité détentrices du savoir et investies du pouvoir d'énoncer des vérités, d'établir la morale, d'instruire, de l'autre, les subordonnés, souvent complices, plus rarement victimes innocentes ou héroïques, le narrateur étant quant à lui menacé, victime éventuelle, complice en tout cas. Le wenyan de la préface est ainsi la langue de l'institution cannibale et conservatrice. Le baihua en cours de création, langue à la première personne de l'individu lucide, lui permet une lecture "culturaliste », considérant la société comme régie par des principes structurels intemporels, qui dévoile (kanpo) la dimension de pouvoir du langage et des textes, leur non-dit et leur hypocrisie. Le bilinguisme de la nouvelle crée une double énonciation (éditeur/diariste), véhicule de valeurs contraires, et une duplicité herméneutique dans la lecture de la nouvelle. Ce dispositif crée une double réception par l'institution de deux narrataires différents renvoyant au même lecteur : le lecteur putatif de la nouvelle est appelé à considérer le diariste comme coupable (il a été cannibale, et s'il a guéri, il est resté tel) et victime (l'éditeur ment, et le « fou » a été mangé), fou et sain d'esprit. Appelé à se reconnaître dans le fou et à apprendre à lire avec lui, il n'en est pas moins le premier destinataire de la préface, qui l'intègre de fait dans la communauté des médecins nécrophages et lettrés cannibales.

La nouvelle, en effet, est présentée par l'éditeur comme « contribution à la recherche médicale » (yijia yanjiu). L'éditeur, le diariste et son frère sont manifestement membres de l'élite formée aux «nouvelles études » (xinxue) réputées modernes. La médecine aussi bien « chinoise » du médecin bourreau que moderne importée du narrataire médecin, est science du cannibalisme. Autrement dit, la science, discours de connaissance du corps, impuissante à produire l'événement singulier de la prise de conscience, est complice de la circularité cannibale. La nouvelle n'oppose donc pas le moderne au traditionnel, l'Occident à la Chine, ni l'individu innocent à la société corrompue. Elle appelle à une conversion éthique de l'esprit, un changement de langue et de lecture, qui doit être allégorique. Lu Xun métaphorise le discours et la représentation de la science. Il s'approprie le discours de la théorie de l'évolution, transformée en une généalogie phylogénétique du cannibalisme, de son acceptation ou de son refus :

Les premiers hommes qui vivaient encore à l'état sauvage ont sans doute mangé de la chair humaine. Par la suite, comme leur mentalité avait évolué, certains y ont renoncé, et par désir de s'amender, sont devenus des hommes dignes de ce nom. Mais d'autres en mangent encore. C'est comme les larves ; certaines sont devenues des poissons, des oiseaux ou des singes, et, pour finir, des hommes ; d'autres n'ont pas voulu s'amender et sont restées des larves. Les hommes qui mangent encore 
leurs semblables doivent se sentir bien honteux (cankui) en se comparant à ceux qui n'en mangent plus. Infiniment plus honteux, je le crains, que les larves vis-à-vis des singes ${ }^{93}$.

Le véritable évolutionnisme est une histoire de la morale, dont le moteur est le choix éthique ${ }^{94}$ du refus de la « prédation », grâce à la logique du Fou qui établit un lien entre les faits qu'il réunit ${ }^{95}$. La science n'est pas rejetée, car le principe de l'évolution est assimilé (malgré quelques libertés vésaniques avec le schéma standard). Ce n'est pas non plus une simple transposition du discours du surhomme de Nietzsche dans la paraphrase transparente qui en est donnée $\mathrm{ici}^{96}$. Le « fou » est trop humain pour être un Zarathoustra. Cette appropriation métaphorique du paradigme scientifique du biologique vers le moral, de "l'homme sauvage » (yemande ren) aux « hommes authentiques » (zhende ren), est décrite dans la « Préface » comme passage du corps à l'esprit, des sciences aux lettres. L'écriture diariste fictive constitue la véritable recherche médicale. La nouvelle est fondatrice en ce qu'elle inaugure dans la Nouvelle littérature le « genre » de l'écriture de la maladie, de l'écriture clinique ${ }^{97}$, comme véritable médecine, tandis que l'auteur se représente en médecin nosographe de la culture. Celui-ci relève des symptômes, établit un diagnostic, définit une topique des maux de l'esprit. Or, ceux-ci sont représentés par des motifs corporels allégoriques.

Dans « Le journal d'un fou », le corps ne fait l'objet d'aucune description particulière. Il est évoqué comme l'interface permettant à la société d'asseoir son emprise sur l'individu. Il n'est que la pâture de la scopie cannibale et des « crocs » des autres en un monde où homo homini lupus. Tout juste bon à palper par le médecin maître queux, dont la fonction est d'engraisser les victimes. Le corps humain qui, pour le fou renversant les causalités, semble le fruit d'une évolution morale, est un aliment. Le destin du diariste est celui de l'individu dans un monde cannibale : il a mangé et sera mangé. Les corps ne s'appartiennent pas, ils sont menacés et aliénés. Les rapports humains sont une affaire d'estomac : celui qui accepte la loi commune mange, celui qui comme

93. Tr. Vallette-Hémery $1991: 20$. Lu 2006e : 17.

94. Itô 2005 : 107. Cette dimension éthique est nouvelle pour Lu Xun et n'apparaît pas dans de précédentes mentions du schéma évolutionniste (Lu 2006b : 31).

95. Quignard $1997: 37$.

96. Nietzsche 1999 : 22. Voir aussi Findeisen $1997: 84-88$; Galik 1986 : 26-27. Lu Xun a traduit, partiellement en wenyan puis entièrement en baihua le « Prologue de Zarathoustra» entre 1918 et 1920 (Findeisen $2001: 778$ et $1997: 86$ ).

97. Vuilleumier $2010: 214-236$. Il faut réserver une place à part pour les références de Lu Xun à sa propre maladie dans certains textes, et surtout, son Journal. 
le fou la refuse, vomit. L'activité «phagique » institue le corps propre comme « interface » entre le sujet et son environnement humain, devenu comestible et assimilable. Ce thème regroupe une série de motifs chez Lu Xun : cannibalisme, regards voraces, autophagie. Le banquet est une allégorie de la Chine qui, tel Cronos, dévore sa jeunesse ${ }^{98}$, de la société chinoise représentée comme une tablée, instituant autour d'elle un immuable rituel de partage inhumain. Et la conscience de cette complicité, autant que celle de la menace, est une souffrance ${ }^{99}$.

\section{Le corps victime de la lettre et de la Loi}

\section{Le corps malade de la culture}

L'aliénation du sujet s'exprime également par le motif du corps démembré. Toute exécution capitale se lit comme pratique arriérée cannibale cause d'apathie chez les spectateurs complices, et de traumatisme pour le témoin. Dans «Le remède » (« Yao », 1919), les parents d'un enfant tuberculeux lui donnent un petit pain trempé dans le sang d'un homme exécuté. Dans « Bénédiction » («Zhufu », 1924), l'héroïne, ostracisée par les maîtres qu'elle sert, passant pour dotée d'une influence néfaste car deux fois veuve, est tenue à l'écart des rituels communs. Elle craint d'être découpée en deux par le roi des Enfers une fois morte, pour être répartie à part égale entre ses maris défunts. Le démembrement est métaphore de la condition pitoyable de cette femme paysanne pauvre, aliénée par la superstition, la religion, et le patriarcat : dans la littérature du 4 Mai, la paysanne est la figure par excellence de l'individu exploité. Le corps féminin depuis au moins les discours réformiste des années 1860 véhicule un discours critique ou normatif sur la collectivité. Dans «Ce que je pense des martyres de la vertu» (« Wo zhi jielie guan», 1918), la femme est interdite de parole, «son corps (tizhi) est déformé, comme son esprit (jingshen) ${ }^{100}$. Le corps est déformé, dévitalisé, rigide, « comme du bois », la peau marquée comme des «écorces de pin », comme c'est le cas dans «Pays natal » (« Guxiang », 1921) du cousin paysan du narrateur, écrasé par les éléments, les autorités, les militaires et son propre conservatisme. L'inertie,

98. Davies $2013: 61$.

99. S'agit-il, selon les termes du Fou, de « honte » (cankui), un sentiment proche de celui de l'humiliation collective, ou de culpabilité, partagée à titre individuel ? Voir Tsuneki 2006 : 346-347.

100. Lu 2006d : 125. 
comme l'apathie complice du spectateur, empêche l'action, la vie. Dans « Pays natal », le corps raide de la «Vénus au tofu » («Doufu Xi Shi »), à cause de ses pieds bandés, à lire comme signe de la société arriérée, est grotesque. Le motif du handicap physique entre dans cette catégorie : le personnage de Kong Yiji, dans la nouvelle éponyme de 1919 est un lettré raté, condamné à se traîner par terre à cause de ses jambes brisées ${ }^{101}$. La culture et l'éducation classiques sont les responsables indirects de la paralysie, car il est incapable de se faire entendre ni faire autre chose que de recopier des textes : le corps est victime des textes classiques ${ }^{102}$. Le nom même du personnage, renvoyant à celui de Confucius (Kongzi), incarne des lignes d'écriture dénuées de sens à réaliser par les enfants à partir de modèles d'écriture.

\section{Techniques du corps, norme et morale}

L'individu est soumis à différentes techniques du corps qui l'altèrent. Comme la médecine, elles sont « traditionnelles » autant que « modernes », matérielles et aliénantes. La première des techniques trompeuses est la médecine elle-même. La médecine chinoise absconse appauvrit les malades avant de les tuer : c'est le cas dans «La maladie de mon père » («Fuqinde bing », 1926), qui reprend un autre mythème traumatisant luxunien, celui de la maladie du père, affaibli par les remèdes coûteux et inefficaces prescrits par un médecin chinois, responsables d'avoir ruiné la famille ${ }^{103}$. Cet épisode est par ailleurs associé à un sentiment de honte lié à la pauvreté (relative) et la déchéance sociale et paternelle. Dans « Je passe donc de ma moustache à mes dents » («Cong huxu shuodao yachi », 1925), Lu Xun raconte comment en Chine son affection dentaire ne peut être traitée, et lui vaut au contraire une réprimande d'ordre moral, le rendant responsable de sa maladie ${ }^{104}$. Il prétend avoir été guéri dès son arrivée au Japon par la médecine moderne. Le récit de cette guérison vise surtout à disqualifier la médecine et le moralisme anciens. Pourtant, si la médecine pratiquée au Japon est métaphore de la modernité, cette dernière n'est pas exempte de critiques. On pourrait citer « l'hygiène » (weisheng), l'une des modalités pratiques du discours normatif de la modernité à la période républicaine, action de dressage du corps. Lu Xun dénonce

101. Le motif de l'homme affaibli, féminisé, face à la femme émancipée ou forte hante la littérature chinoise moderne.

102. On lui a brisé les jambes en punition d'un vol commis.

103. Leo Lee Ou-fan a d'ailleurs proposé de voir dans le parcours de Lu Xun la volonté de restaurer l'honneur de son père (Lee 1987).

104. Von Kowallis 1996 : 20. 
indirectement son hypocrisie, qui masque des motivations réactionnaires, et son inefficacité ${ }^{105}$.

Les châtiments représentent un développement extrême des techniques anciennes. Lu Xun, dans " Propos variés après ma maladie » (« Binghou zatan », 1935), voit en Chine un retard de la science et de la médecine, exception faite de la pratique des supplices, demandant une maitrise et une bonne connaissance empirique, en particulier dans le cas des peines de "castration » (gongxing, youbi). Les « supplices » (nüexing) sont une déviation de la «médecine » (yishu) et de "l'anatomie » (jiepou) ${ }^{106}$, développés uniquement comme techniques de punition. De même que le fou découvrait dans les Classiquesconfucéens et les annales officielles une histoire du cannibalisme, $\mathrm{Lu}$ Xun malade trouve dans les « notes » (biji) et « histoires non officielles » (yeshi) confirmation que l'histoire chinoise est celle des supplices : les textes anciens sont une description méthodique de la violence, ceux-là métaphores de celle-ci et réciproquement, ce dont témoigne les citations de récits classiques «d'écorchements » (bopi) qui ponctuent ses considérations ${ }^{107}$.

Parmi les autres techniques anciennes, les arts martiaux et la pratique de la transe maintiennent leurs adeptes en état d'apathie : dans « Le Savon », le jeune écolier pratique la « Boxe des huit trigrammes » (baguaquan) dans l'obscurité ; dans « Hors des passes », Laozi est pareil à du «bois mort et de la cendre éteinte », à un « morceau de bois stupide » (dai mutou). Ces techniques ridiculisées renvoient à la littérature populaire (la Nouvelle littérature cherchant à se démarquer de "l'ancienne littérature »), à la vogue des «arts (martiaux) nationaux » (guoshu), tout autant que des livres de méditation. Car ces techniques expriment une volonté de retour en arrière, de corporalité et d'immobilisme.

\section{Le corps comme réalité sociale aliénante}

Le corps offre une prise au pouvoir politique quand, au risque de la décapitation, il fait, sans pouvoir s'y soustraire, gage d'allégeance personnelle à un régime ou à une faction. Le motif des cheveux ou des moustaches l'exprime : avoir les cheveux courts dans les années 1900 est un crime à

105. Voir «Le savon» (Feizao, 1924), Lu 2006f : 41-53; Lu 2004 : 61-74. Dans « Hors des passes » (Chu guan, 1936), Lu Xun ironise sur la mode du discours hygiéniste, repris par un douanier (Lu 2007c : 107 ; Lu et Li 1988: 140).

106. Lu 2007b : 168-169.

107. Lu 2007b : 168-170. Il ne s'agit pas de «la mort lente» (lingchi 凌遲), qu'il mentionne (ibid: 171). 
l'égard des Mandchous qui avaient imposé le front rasé et la natte aux hommes chinois. En revanche, de porter la natte mandchoue ${ }^{108}$ est un crime à l'égard des Taiping (1851-1864) ${ }^{109}$. De même, une moustache assigne immédiatement une identité à son porteur : Lu Xun raconte comment un pays a refusé de le reconnaître comme Chinois, à cause de sa moustache « japonaise » (« De ma moustache »). Le corps matérialise la personne dans sa dimension sociale, l'ouvre à la menace. Aliénant par nature, il fait l'objet d'un rejet radical. Dans «Les adieux de l'ombre » (« Yingde gaobie », 1924), «l'ombre » (ying) du dormeur - son âme ou son esprit - veut quitter son corps matériel, pour une existence solipsiste. La mention du corps dans le texte est réduite au minimum, à une main (symbole d'action) « gris cendre » inconsistante ${ }^{110}$.

\section{Le corps comme lieu d'écriture de la Loi}

Le corps est aussi métaphore de l'écriture, transformé en signe de la norme destructrice du Père. Il souffre avant tout de la «piété filiale » (xiao), dont le cannibalisme est la métaphore hyperbolique. Le corps est la projection de la dimension sociale de l'individu pris dans un réseau d'obligations éthiques envers les aînés. Selon le Classique de la piété filiale (Xiaojing), le corps appartient aux parents, ce qui implique divers devoirs, comme de leur assurer une postérité (Mencius), ou s'ils sont malades, d'aller jusqu'à leur donner à manger un morceau de son corps ${ }^{111}$. La piété filiale caractérise le « confucianisme », au même titre que les injonctions voraces à la morale ( "Journal d'un fou »). Dans Les Vingt-quatre images de la piété filiale (Ershisi xiao tu, 1926) ${ }^{112}$, Lu Xun désigne la piété filiale comme instrument des «confucianistes » (ruzhe) pour terroriser les enfants. Cette morale est transmise par les livres d'enfants illustrés : le texte s'ouvre sur une «imprécation » lancée par Lu Xun contre les moralistes contempteurs du baihua ${ }^{113}$. La question de la langue est omniprésente : comment agir sur la représentation littéraire du corps pour empêcher la reproduction de la violence par sa représentation. Le corps est la lettre de l'ordre, qui rend possible celui-ci.

108. Porter la natte est un symbole d'humiliation nationale et raciale. Voir Lu 2007b : 191.

109. Voir « Histoire de cheveux » (« Toufade gushi », 1920), Lu 2006e : 49-55; Lu 2010 : 61-66.

110. Lu 2006c : 7 ; Vuilleumier 2006.

111. Voir «Quels pères devons-nous être aujourd'hui », Lu 2006d : 135, 140 ; «La véritable histoire d'A Q » («A Q zhengzhuan », 1921-1922), Lu 2006e : 90.

112. Vingt-quatre images de la piété filiale est un ouvrage de morale classique destiné aux enfants.

113. Lu 2007e : 24 . 
La culture chinoise dénature les corps réels par l'action du wenyan, réduisant ceux-ci au rôle de signe de cette tradition. Dans «La réparation du ciel » («Bu tian », 1922) la géante Nüwa, déesse créatrice des hommes, est prise à partie par un censeur confucianiste campé entre ses jambes lui reprochant en wenyan sa nudité. Plus tard, Nüwa morte épuisée, son corps est pris d'assaut par des armées opposées, plantant des drapeaux de caractères archaïques, afin de prendre possession de son corps et d'en tirer légitimité. Cette critique rousseauiste de la culture et de l'histoire lettrée représente dans ce récit en baihua la mort du corps nu et naturel, tué par l'évolution de la langue des premiers hommes, de simples onomatopées en lettres latines (négation du caractère chinois) au wenyan moral et inaudible de leurs descendants. La «Boxe des Huit trigrammes » dans «Le savon » forme la même imposition de caractères sur le corps : les trigrammes, origine fantasmatique des caractères ${ }^{114}$, sont le signe de la culture lettrée, cherchant à maintenir une totalité «symbolique » entre le corps et la lettre, un tatouage du sens. Dans « Souvenirs du passé » ( « Huaijiu », 1913), nouvelle en wenyan, le narrateur rappelle ses souvenirs de jeune écolier : les caractères sont perçus visuellement comme indistincts du maitre ${ }^{115}$, faisant corps avec lui. La pratique classique de l'écriture, de la lecture et de la transe psalmodiée sont des techniques du corps qui en prennent possession.

\section{La destruction de la représentation complice du corps}

La représentation physiologique pourrait contrer la représentation « classique » du corps. Dans « Je passe donc de ma moustache à mes dents », Lu Xun parle volontairement du «bas » (xiati) et après avoir revendiqué le droit d'évoquer ses «poils » de moustache ( De ma moustache », « Shuo huxu », 1924), mentionne ses dents pour en arriver à décrire le trajet de l'alimentation, de l'ingestion à l'excrétion, recourant au lexique anatomique ${ }^{116}$. C'est l'une des rares descriptions du corps chez lui, comme on en trouve encore dans « Propos après ma maladie », où il décrit les symptômes de sa maladie :

114. Lu Xun donne comme origine à l'apparition de la langue, dans une perspective assez rousseauiste (tout autant que traditionnelle), l'expression inarticulée par le corps et les cris, le besoin d'expression poétique au contact du monde, puis reprend les lieux classiques sur l'invention de l'écriture, des cordes nouées aux trigrammes et hexagrammes, qui auraient précédé les caractères d'écriture (Lu 2007f : 1-2).

115. Lu 2007a : 2 ; Lyell $1976: 317$.

116. Lu 2006d : 259 . 
l'objectif est de choquer, de se montrer « vulgaire » $(s u)^{117}$, et d'opposer à une conception holistique et analogique, une représentation matérielle et sans implication morale du corps, désacralisante et scatologique. Il ne s'agit pas de décrire le corps, même si on peut considérer ce souci du corps individuel et maladif comme expression modernisée du souci de soi des lettrés Wei Jin, avec lesquels Lu Xun tisse des liens profonds ${ }^{118}$. Sur ce point, Lu Xun se place dans la perspective du 4 Mai et de la Nouvelle culture, qui revendique la modernité de la représentation scientifique du corps.

L'exemple le plus spectaculaire de cette entreprise de destruction symbolique de la représentation du corps se trouve dans «Vengeance » («Fuchou », 1924). Ce poème en prose décrit l'imminence d'une lutte entre deux personnages non identifiés ${ }^{119}$ qui n'aura pas lieu, et la réaction de dépit de la part de spectateurs qui escomptaient sang et violence. La première partie du texte est une rêverie de dissection morbide, sur le modèle d'une séance d'anatomie, sadique car associant plaisir et meurtre. L'ironie du texte cible l'attente suggérée du narrataire, représenté par les badauds censés espérer que les personnages «s'embrassent ou se poignardent» (jiangyao yongbao, jiangyao shalu $)^{120}$. Le lecteur, trompé par le titre, est frustré dans son attente de description d'une vengeance. Lu Xun combat l'esprit de vengeance impliquant la représentation de la violence et du supplice, qui seraient véhiculés par la littérature chinoise ancienne (tel Au Bord de l'eau) ${ }^{121}$. Hu Shi (1891-1962) et Zhou Zuoren (1885-1967) ont été parmi les premiers à dénoncer celle-ci comme facteur d'arriération reproduisant la pensée ancienne. Les badauds vermiculaires sont cousins des « tarentules » de Zarathoustra, le pourfendeur de la morale du ressentiment qui travestit en « justice » le désir de vengeance ${ }^{122}$. La représentation littéraire chinoise du corps appellerait à la violence. C'est donc l'image du corps que Lu Xun découpe par la chirurgie du texte : cette destruction de la «peau de pêche » (taohong sede pifu), épithète stéréotypée classique, effectue symboliquement une «"vengeance" sur l'ancien système de représentation ${ }^{123} »$. Pourtant, le texte ne procède pas à son remplacement intégral par une description anatomique « moderne ». L'objectif reste destructeur. À

117. Lu 2007b : 168 .

118. Vuilleumier 2011a.

119. Lu Xun dit ailleurs qu'il s'agit d'un homme et d'une femme (Lu 2006c : 15). Mais le texte ne le dit pas. La question des genres n'est pas importante ici.

120. Lu 2006c :14-15; Lu 1975.

121. Sur le supplice comme métaphore de la littérature, voir Veg $2005: 272$.

122. Nietzsche 1999: 128-131.

123. Heinrich 2002 : 177. 
travers le corps, la vengeance porte sur la culture chinoise. Malgré le refus explicite de reproduire la violence en la représentant, on peut se demander si le texte n'exprime pas thématiquement ce qu'il nie discursivement, à savoir le «mal » ${ }^{124}$. Le texte est structuré par l'image du mouvement des insectes ${ }^{125}$, l'unité d'Éros et Thanatos : le thème d'un flux vital continu, indifférencié et toujours renouvelé. Cette destruction, même si elle vise la représentation du corps, reste d'une grande violence symbolique ${ }^{126}$.

\section{La cicatrice}

Le passé culturel et individuel, perpétué dans la Chine moderne, aliène les individus. La cicatrice en signale le traumatisme sur le corps. Les personnages de A Q et de Maître Gao en portent une sur la tête : pour le premier, elle est le signe de son «A-Qisme ${ }^{127}$ », tare morale qui lui fait ressentir une victoire imaginaire et masochiste en consolation de sa condition abjecte - la cicatrice s'illumine et provoque les rires. Quant à celle de Maître Gao, elle matérialise son malaise face au regard des autres ainsi que son conservatisme ${ }^{128}$. Situer la cicatrice sur le visage la désigne comme blessure narcissique, et elle vise ou dévoile la «face» $(\text { mianzi })^{129}$. Les signes corporels forment l'allégorie de la «nature nationale » (minzuxing ou guominxing) supposée des Chinois : Lu Xun a lu Chinese Characteristics d'Arthur Smith pendant ses années japonaises ${ }^{130}$. Les motifs corporels disent les tares spirituelles et culturelles, autant de

124. Voir Bataille $2004: 21,24$ et la «narration préhumaine » (Quignard $1997: 142$ ) à l'orée de tout texte.

125. Il existe un rapport intertextuel direct avec «Une charogne» (Heinrich $2002: 181$ ). «Vengeance » est publié dans le numéro 7 de la revue Au fil des mots (Yusi). Or, dans le numéro 3, Xu Zhimo (1897-1931) publie une version du poème de Baudelaire, accompagnée d'une présentation à laquelle Lu Xun réagit de façon très critique dans le numéro 5. Lu Xun critique le symbolisme mystique de Xu Zhimo, dont il reprend l'imaginaire.

126. Est-elle portée contre le corps en tant qu'objet de la piété filiale ou du poids du passé (le Père à travers lui) ? ou en tant qu'obstacle créant de la « discontinuité » dans la « continuité » première ? s'agit-il d'un dispositif de distanciation cathartique pour le lecteur (voir Kaldis $2014: 179-1781$ ) ?

127. La lettre « $Q »$ figure une tête nattée.

128. « Maître Gao » (« Gao lao fuzi », 1925), Lu 2006f : 72-83 ; Lu 2004 : 97-109.

129. La «face » est un besoin de respectabilité hypocrite impliquant une conception hiérarchique et inégalitaire de la société : «À propos de la "face” » («Shuo mianzi », 1934), Lu 2007b : 128-131 ; “"Face” et "Pourboire” » (« Mianzi he menqian », 1923), Lu 2006a : 398-401.

130. Liu 1995 : 45-77. 
maladies congénitales ${ }^{131}$. Les personnages, mais également les narrateurs ( « Pays natal », «Bénédiction », « Kong Yiji ») souffrent du passé. Le narrateur ou Lu Xun tel qu'il se représente, sont tourmentés par les «ténèbres » (hei'an) du passé mêlant fiction et autobiographie ${ }^{132}$. Le mal de dents dont Lu Xun dit avoir guéri au Japon est interprété via l'écriture comme symptôme du malaise culturel de la Chine. Écrire sur la maladie personnelle est prétexte pour parler de la maladie de la culture chinoise («Écrit après ma maladie »), à moins que ce ne soit l'inverse : «J'anatomise souvent les autres, en fait, le plus souvent, c'est moi-même que j'autopsie froidement ${ }^{133}$. »

Le corps, chez Lu Xun, en plus d'être interface sociale, est surface d'écriture. Sa représentation implique des attentes éthiques et normatives qui l'aliènent, l'affaiblissent ou le détruisent. Il s'agit d'empêcher sur le corps le tatouage de l'écriture de la Loi et de sa peine ${ }^{134}$. Lu Xun montre un corps décapité, aliéné ou découpé, contre une représentation « classique »du corps, « distinguée » $(y a)$, mais totalisante et trompeuse ${ }^{135}$. À l'inverse de l'esthétique du tatouage dans laquelle le signe et le sens se rejoignent ${ }^{136}$, il choisit la cicatrice et l'usage allégorique du corps. Ce choix interdit tout pathos.

Le corps, chez Lu Xun, est souffrant : mais il s'agit moins d'une souffrance du corps que d'une souffrance signifiée par la figure du corps. Le corps est absent de la description. Le corps propre, lorsqu'il est brièvement dit, est certes toujours malade et souffrant : mais il n'est pas individualisé. Il est transformé en allégorie du corps malade de la nation, et plus abstraitement encore, de l'esprit, de la culture (tenant à distance le corps propre). Les esprits sont empoisonnés par un mal héréditaire commun, qui hante les consciences et se marque sur les corps. Le corps, dont la représentation est réduite à des motifs corporels, apparaît comme allégorie, nationale et esthétique. Esthétique, elle est rupture du corps avec l'ordre moral du monde, de la lettre avec sa capacité d'expression : le corps vidé de toute « spiritualisation ${ }^{137}$ » est le corps chinois désemparé, à la recherche d'une nouvelle voix. La cicatrice qui signale la perte d'intégrité du corps, une rupture définitive avec un système symbolique aliénant, est le propre de l'allégorie comme refus de l'utopie ouverte par le corps

131. Lu 2006d : 137.

132. Lu et Jing $2006: 19$.

133. « Écrit après La Tombe » (Lu 2006d :298).

134. Agamben $2015: 25$ (ce qu'illustre la « La colonie pénitentiaire » de Kafka).

135. Les suppliciés « distingués » font de la poésie avant leur exécution : métaphore pour la littérature classique masquant la violence sociale ( $\mathrm{Lu} 2007 \mathrm{~b}: 170$ ).

136. Pour un exemple chez Guo Moruo (1892-1978), voir Vuilleumier 2015.

137. Benjamin $2000: 218$. 
(ici le signe classique) ${ }^{138}$, exprimée par le tatouage totalisant et inclusif. Ceci explique l'usage principalement figuré du corps chez Lu Xun : le corps est une métaphore et ne peut parler pour le corps réel, il établit l'absence du corps et oblige à une distance irréductible avec l'objet de la représentation. À l'inverse de l'image réaliste projetée par diapositive de la décapitation, l'allégorie littéraire des motifs corporels ne peut permettre aucune identification pathétique de la part du lecteur. Lu Xun veut intellectualiser la souffrance, parce qu'il veut en prouver l'origine culturelle et spirituelle. Cette distance se voit également dans le dispositif de mise à distance narratoriale des nouvelles ${ }^{139}$ ou dans la double énonciation du «Journal d'un fou ». La souffrance n'est pas dite directement pour cette raison - en même temps, cette distance crée le plus d'effet. Cette volonté d'intellectualiser le corps se voit dans la métaphorisation de la médecine, science du corps, qui devient nosographie spirituelle et culturelle. Le corps est le signe de la mémoire traumatisée, et la maladie, celle de l'esprit et de l'écrit. La « spiritualisation » du « matériel » constitue de plus un projet d'appropriation de la modernité scientifique importée par les réformistes, ceux-là mêmes qui ont introduit la crainte d'être en retard dans le schéma d'évolution des peuples. Et c'est une "modernité mondialisée », comme le montre notre analyse du discours culturaliste de réforme dans le «Journal d'un fou », où un sujet autonome revendiquant un discours culturaliste veut se placer au centre de la transformation politique de la nation en « retard» par un processus rationnel. Il en assume l'entière responsabilité ${ }^{140}$, pour transformer en culpabilité la honte ressentie face à l'Occident. Pour affirmer une différence avec ce dernier, malgré la nature fondamentalement hybride de son propre discours ${ }^{141}$, puisque même le projet d'édification nationale est mondialisé, il cherche à le spiritualiser, le rendre éthique, pour maintenir un jeu critique à l'intérieur même du discours importé de la « science » et donc du corps et de ses représentations.

Lu Xun refuse les stéréotypes classiques mais ne crée pas de nouvelle représentation littéraire du corps. En de rares endroits, il introduit une vision 《 réaliste », donc "moderne » et « humaniste » du corps, que le 4 Mai appelle à affirmer, programme appliqué par des auteurs comme Guo Moruo

138. Foucault $2014: 15$.

139. Anderson $1990: 32-53$.

140. Ce qui demande au sujet de se maintenir dans le renouvellement permanent d'un instant critique, moderne, impossible à saisir, qui annonce l'avenir et le transforme, «puis[ant] sa normativité en elle-même » (Habermas $2011: 8$ ).

141. Hybride, car il contient des références multiples occidentalistes et aussi indigènes, comme pour le thème de la folie (voir Gao 2007 : 45). 
ou Yu Dafu (1896-1945) ${ }^{142}$. Lu Xun ne suit pas cette construction moderne de la représentation littéraire du corps. Il refuse de reprendre ce processus d'objectivation du corps, qui est renforcé par les discours racialistes et nationalistes : de tels auteurs ont repris dans leur représentation littéraire la fondation biologique des identités (genre, nation, race) dans le corps ${ }^{143}$. Non sans contradiction, s'ajoute chez ceux-ci une conception subjectiviste du corps libidinal et du moi corporel inspiré de la psychanalyse. Lu Xun, lui, se montre « conservateur» dans sa représentation des identités. Les motifs corporels sont utilisés comme symboles pour aborder l'identité, la culture, le politique. L'identité chez lui est non biologique, mais demeurée culturelle, fondée à travers le texte et la mémoire ${ }^{144}$. Pourtant, la représentation du corps de Lu Xun n'est pas dénuée de «modernité ». En témoigne une réception de la psychanalyse freudienne, limitée à quelques textes (ou sous la forme dérivée de l'esthétique du kumen) et surtout, sa conception «évolutionniste » de la vie comme d'un procès continu fait de transformation et lutte, demandant de « remplacer le vieux par le nouveau ». Lu Xun est par contre esthétiquement moderniste dans sa représentation du corps ${ }^{145}$. Ce qui n'exclut pas un certain classicisme. Si dans l'art et la littérature pré-modernes, le corps est «invisible » ${ }^{146}$, habillé, culturel, s'il est évoqué, stéréotypé ou caché par des expressions figées renvoyant à des gestes ou attitudes éthiques et morales, matérialisation d'un éthos, l'absence chez Lu Xun d'individualisation des corps et leur esquisse ${ }^{147}$ perpétuent cette « tradition ». D'ailleurs la maladie comme métaphore n'est en rien nouveau dans l'histoire de la littérature chinoise ${ }^{148}$. Lu Xun ne cherche pas à décrire le corps ni à le libérer de sa fonction de signe, mais à critiquer la culture, l'esprit donc, car le corps, chose du bio-pouvoir, reste fondamentalement métaphore de la matière.

142. Ils introduisent consciemment dans leurs nouvelles des années 1920 et 1930 le paradigme du déterminisme de la conscience et du sujet par «l'instinct »; représentation moderne de la mécanique du corps objet autonome, dont les « muscles » (jinrou), activés par les «nerfs » (shenjing), transmettent les ordres du cerveau.

143. Voir Siary 2014 ; Dikötter 1995.

144. Sur ce point, il y a proximité avec des œuvres telles L'Odyssée de Lao Can (ou Vieux-Débris, Lao Can youji 老殘遊記, 1903-1906), Fleurs dans un océan de péchés (Niehaihua 蕉海花, 1903-1931) ou les nouvelles de Su Manshu (蘇曼殊, 1894-1918).

145. Symboliste, expressionniste, inspiré par les effets du cinéma (voir Hanan 1974 : 89) et de la gravure.

146. Hay 1994.

147. Semanov $1980: 96$ sqq.

148. Tan 2007. 
Ce refus de «moderniser » le corps en littérature est cohérent avec le rejet de la normativité nouvelle. Lu Xun refuse de se rendre complice de ce contrôle au niveau de la représentation du corps : il résiste ainsi aux idéologies du retour en arrière, comme à celles du nationalisme et du scientisme. Le discours de la modernité importée comme discours du corps est transformé en allégorie littéraire $^{149}$. Le paradoxe de Lu Xun est qu'il y parvient dans une langue nouvelle, tout en recourant à des références et procédés classiques : sur ce point, il est autant un renaissant qu'un moderne.

149. La destruction symbolique du corps (similaire à la promotion de la latinisation, latinxua 拉丁化) chez Lu Xun est différente des représentations dualisantes de la Nouvelle littérature des années 1920, opposant à l'âme souffrante le corps déprécié, qui expriment ainsi directement leurs frustrations. Pourtant, Lu Xun n'est pas un « contempteur du corps », même si celui-ci tend à servir surtout « l'esprit » (voir Gao 2007 : 181). Il vise le Körper, non le Leib, insistant avec Nietzsche sur le processus de vie (leben) et le rejet du désir de mort, mais rejetant l'esthétique du corps et du surhomme. Lu Xun combat le corps comme expression figée et obstacle à la vie, pour contrer la souffrance de cette impossibilité à régénérer la voix. Cependant, le corps littéraire, par sa destruction symbolique et sa mise à distance comme signe, laisse soupçonner une ultime aliénation du corps. Car, si le corps individuel comme « lieu ou scène de la manifestation [d'un] trouble » est « premier signifiant mis en œuvre par le langage » (Starobinski $1981: 273$ ), comment comprendre qu'il soit tu ? C'est peutêtre la dernière souffrance, celle du corps muet, obligé de demeurer un signe statique interdit de parole individuelle, au nom de la dénonciation du silence collectif et du refus de l'épanchement. 


\section{BIBLIOGRAPHIE}

AdMussen, Nick (2009). «A Music for Baihua : Lu Xun's Wild Grass and "A Good Story” ». Chinese Literature : Essays, Articles, Reviews, vol. 31 : 1-22.

AgAmBEn, Giorgio et RuEFF, Martin (trad.) (2015). Le Feu et le récit. Paris, Rivages.

ANDERSON, Marston (1990). The Limits of Realism. Chinese Fiction in the Revolutionary Period. Berkeley, University of California Press.

Assoun, Paul-Laurent (2009). Corps et symptôme. Leçons de psychanalyse. Paris, Anthropos.

BATAILlE, Georges (2004). La Littérature et le mal. Paris, Gallimard.

BENJAMIn, Walter et Muller, Sybille (trad.) (2000). Origine du drame baroque allemand. Paris, Flammarion.

CAO, Juren 曹聚仁 (2006). Lu Xun pingzhuan 魯迅評傳. Shanghai, Dongfang chuban zhongxin.

CHARTIER, Roger (2009). Au bord de la falaise, L'histoire entre certitudes et inquiétude. Paris, Albin Michel.

CHEN, Pingyuan 陳平原 (1998). Zhongguo xiandai xueshu zhi jianli 中國現代學術之 建立. Pékin, Beijing daxue chubanshe.

CHOw, Rey (1995). Primitive Passions. Visuality, Sexuality, Ethnography, and Contemporary Chinese Cinema. New York, Columbia University Press.

DAVIES, Gloria (2013). Lu Xun's Revolution, Writing in a Time of Violence. Harvard University Press.

DIKÖTTER, Frank (1995). Sex, Culture and Modernity in China, Medical Science and the Construction of Modern Identities in the Early Republican Period. Londres, Hurst \& Co.

DIRLIK, Arif (2010). «Asian Modernities in the Perspective of Global Modernity ». In CHU, Yiu-wai et MAN, Eva Kit-wah (dir.), Contemporary Asian Modernities, Transnationality, Interculturality, and Hybridity. Berne, Peter Lang : 27-54.

FInDEISEN, Raoul D. (2001). Lu Xun (1881-1936). Texte, Chronik, Bilder, Dokumente. Bâle, Stroemfeld.

FINDEISEN, Raoul D. (1997). «The Burden of Culture : Glimpses at the Literary Reception of Nietzsche in China ». Asian and African Studies, vol. 6, $\mathrm{n}^{\circ} 1$ : 76-91.

FouCAUlt, Michel (2014). Le Corps utopique, Les Hétérotopies. Fécamp, Lignes.

FRAN, Martin et HEINRICH, Larissa (dir.) (2006). Embodied Modernities, Corporeality, Representation, and Chinese Culture. Honolulu, University of Hawai'i Press.

GALIK, Marian (1986). Milestones in Sino-Western Literary Confrontations (18981979). Wiesbaden, Harrassowitz.

GAO, Junlin 高俊林 (2007). Xiandai wenren yu “Wei Jin fengdu” 現代文人與“魏晉風 度'. Zhengzhou, Hennan renmin wenxue chubanshe.

GAO, Yuanbao 郜元寶 (2007). Lu Xun liu jiang 魯迅六講. Pékin, Beijing daxue chubanshe.

GERNET, Jacques (1995). L'Intelligence de la Chine. Le social et le mental. Paris, Gallimard. 
HABERMAS, Jürgen (2011). Le Discours philosophique de la modernité. Paris, Gallimard.

Hanan, Patrick (1974). « The Technique of Lu Hsün's Fiction ». Harvard Journal of Asiatic Studies, vol. 34 : 53-96.

HAY, John (1994). « The Body Invisible in Chinese Art? ». In ZITO, Angela, BARLOW, Tani E. (dir.), Body, Subject and Power in China. Chicago, The University of Chicago Press : 42-77.

HEINRICH, Larissa (2002). The Pathological Body : Science, Race and Literary Realism in China, 1770-1930. Ann Arbor, UMI.

Heubel, Fabian (2015). "Chinesische Philosophie im Umbruch. Über einige Konsequenzen hybrider Modernisierung ». Article à paraître communiqué par son auteur.

HUANG, Jinlin 黃金鱗 (2006). Lishi, shenti, guojia. Jindai Zhongguode shenti xingcheng (1895-1937) 歷史、身體、國家：近代中國的身體形 (1895-1937). Pékin, Xinxing chubanshe.

ITÔ, Toramaru 伊藤虎丸, SUN, Meng 孙猛 XU, Jiang 徐江, LIDONGMU 李冬木 (trad.) (2005). Lu Xun, chuangzaoshe yu ribenwenxue - Zhong Ri jinxiandai bijiao wenxue chutan 魯迅、創造社與日本文學一中日近現代比較文學初探. Pékin, Beijing daxue chubanshe.

JAMESON, Fredric (automne 1986). « Third-World Literature in the Era of Multinational Capitalism ». Social Text, $\mathrm{n}^{\circ} 15: 65-88$.

Kaldis, Nicholas A. (2014). The Chinese Prose Poem, A Study of Lu Xun's Wild Grass (Yecao). Amherst, New York, Cambria Press.

KEMPF, Roger (1968). Sur le corps romanesque. Paris, Seuil.

VonKowallis, Jon Eugene (2013). « Translating Lu Xun's Mâra : Determining the "Source" Text, the "Spirit" versus "Letter" Dilemma and Other Philosophical Conundrums ». Frontiers of Literary Studies in China, vol. 7, n ${ }^{\circ} 3$ : 422-440.

Von KowalLIS, Jon Eugene (1996). The Lyrical Lu Xun. Honolulu : University of Hawai'i Press.

Lee, Leo Ou-fan (1987), Voices from the Iron House, A Study of Lu Xun. Bloomington, Indiana University Press.

LIU, LYDIA (1995). Translingual Practice, Literature, National Culture, and Translated Modernity, China, 1900-1937. Stanford, Stanford University Press.

Lou, Yulie (dir.) et KNEChtGes, David R. (trad.) (2012). The History of Chinese Civilization. Volume IV Late Ming and Qing Dynasties (1525-1911). Cambridge, Cambridge University Press.

LU Xun et VON KowALLIS, Jon Eugen (trad.) (2011). «Toward a Refutation of Malevolent Voices ». Boundary 2, vol. 38, n 2 : 39-62.

LU Xun et VEG, Sebastian (trad.) (2010). Cris. Paris, Rue d'Ulm.

LU Xun 魯迅 (2008). Lu Xun yiwen quanji 魯迅譯文全集. Fuzhou : Fujian jiaoyu chubanshe, t. $2: 223-295$.

LU Xun 魯迅 (2007a). Jiwai ji shiyi 集外集拾遺 [1938]. Pékin, Renmin wenxue chubanshe. 
LU Xun 魯迅 (2007b). Qiejieting zawen 且介亭雜文 [1937]. Pékin, Renmin wenxue chubanshe.

LU Xun 魯迅 (2007c). Gushi xinbian 故事新編 [1936]. Pékin, Renmin wenxue chubanshe.

LU Xun 魯迅 (2007d). Jiwai ji 集外集 [1935]. Pékin, Renmin wenxue chubanshe.

LU Xun 魯迅 (2007e). Zhao hua xi shi 朝花夕拾 [1928]. Pékin, Renmin wenxue chubanshe.

LU Xun 魯迅 (2007f). Han wenxueshi gangyao 漢文學史綱要 [1926]. Pékin, Renmin wenxue chubanshe.

LU Xun 魯迅 et JiNG, Song 景宋 (2006). Liangdi shu 兩地書. Pékin, Renmin wenxue chubanshe.

LU Xun 魯迅 (2006). Qiejieting zawenmobian 且介亭雜文末編 [1937]. Pékin, Renmin wenxue chubanshe.

LU Xun 魯迅 (2006a). Lu Xun quanji bu yi 魯迅全集補遺. Tianjin, Tianjin renmin chubanshe.

LU Xun 魯迅 (2006b). Jiwai ji shiyi bubian 集外集拾遺補編 [1938]. Pékin, Renmin wenxue chubanshe.

LU Xun 魯迅 (2006c). Yecao 野草[1927]. Pékin, Renmin wenxue chubanshe.

LU Xun 魯迅 (2006d). Fen 墳 [1927]. Pékin, Renmin wenxue chubanshe.

LU Xun 魯迅 (2006e). Nahan 呐喊 [1923]. Pékin, Renmin wenxue chubanshe.

LU Xun 魯迅 (2006f). Panghuang 徬得 [1926]. Pékin, Renmin wenxue chubanshe.

LU Xun 魯迅 et Sebastian, Veg (trad.) (2004). Errances. Paris, Rue d'Ulm.

LU Xun 魯迅 et LI, Tche-houa (1988). Contes anciens à notre manière. Paris, Gallimard.

LU Xun et LOI, Michelle (dir.) (trad.) (1981). La Tombe. Paris, Acropole.

LU Xun et JULLIEN, François (trad.) (1976). Fleurs du matin cueillies le soir. Lausanne : Eibel.

LU Xun et LEYS, Simon (trad.) (1975). La Mauvaise herbe. Paris, 10/18.

LU Xun 魯迅 (1973). Sanxianji 三閒集. Pékin, Renmin wenxue chubanshe.

LYELL, William A. Jr. (1976). Lu Hsün's Vision of Reality. Berkeley, University of California Press.

McDougall, Bonnie S. (1971). The Introduction of Western Literary Theories into Modern China, 1919-1925. Tôkyô, The Centre for East Asian Cultural Studies.

NiETZSCHE, Friedrich et DE GANDILlaC, Maurice (trad.) (1999). Ainsi parlait Zarathoustra. Paris, Gallimard.

Pollard, David E. (2002). The True Story of Lu Xun. Hong Kong, The Chinese University Press.

QIAN, Liqun 錢理群 (2004). Yu Lu Xun xiangyu 與魯迅相遇. Pékin, Sanlian shudian. QuignaRd, Pascal (1997). Rhétorique spéculative. Paris, Gallimard.

RICHARD, Jean-Pierre (2010). Microlectures. Paris, Seuil.

RICEUR, Paul (1998). Du texte à l'action, Essais d'herméneutique II. Paris, Seuil.

RousSET, Jean (1995). Forme et signification. Essai sur les structures littéraires de Corneille à Claudel. Paris, Corti.

Semanov, Vladimir I. et AlBer, Charles J. (trad.) (1980). Lu Hsün and his Predessors. New York, M. E. Sharpe. 
SIARY, Gérard (2014). « The Reception of the Idea of Race in East Asia ». In BANCEL, Nicolas, DAVID, Thomas et RICHARD, Dominic (dir.), The Invention of Race : Scientific and Popular Representations. New York, Routledge : 145-157.

STAROBINSKI, Jean (1981). « Brève histoire de la conscience du corps ». Revue française de psychanalyse, vol. 45, $\mathrm{n}^{\circ} 2$ (1981) : 261-279.

STAROBINSKI, Jean (1978). L'Eil vivant II, La relation critique. Paris, Gallimard.

SuN, Yushi 孫玉石 (2007). Yecao yanjiu 《野草》研究. Pékin, Beijing daxue chubanshe.

TAN, Guanghui 譚光輝 (2007), Zhengzhuangde zhengzhuang-jibing yinyu yu Zhongguo xiandai xiaoshuo 症狀的症狀：疾病隱喻與中國現代小說. Pékin, Zhongguo shehui kexue chubanshe.

TSUNEKI, Maruo 丸尾常喜 et Qin, Gong 秦弓 (trad.) (2006). “Ren” yu “gui” de jiuge -Lu Xun xiaoshuo lunxi 人與鬼的紅葛 : 魯迅小說論析. Pékin, Renmin wenxue chubanshe.

VALLETTE-HÉMERY, Martine (1991). Treize récits chinois 1918-1949. Picquier.

VEG, Sebastian (2010). « Démocratie, anarchisme et révolution littéraire dans la Chine du 4 Mai ». Études littéraires, vol. 41, n $3: 87-102$.

VEG, Sebastian (2005). "Lu Xun et la cruauté des "supplices chinois" ». In DÉTRIE, Muriel et LEIVA, Antonio Dominguez (dir.), Le Supplice oriental dans la littérature et les arts. Neuilly-les-Dijon, Les éditions du Murmure : 271-286.

VUILLEUMIER, Victor (2015, à paraître). «Shentide zaixian, yixue yu jiepou : 20-30 niandai Guo Moruo xiaoshuoli de yizhong hanhude taidu»身體的再現、醫 學與解剖：20與30年代郭沫若小說里的一種含混的態度. Zhongguo xiandai wenxue yanjiu luncong 中國現代文學研究論叢, vol. 10, $\mathrm{n}^{\circ} 1$.

VuILLEUMIER, Victor (2011a). «Drunk, and Drug Addicted Wei Jin Literati in Modern Chinese Literature : Li Tuozhi's "Feigned Madness", Lu Xun, and the Traditional Spirit of the Intellectual Furioso ». In RoskeR, Jana S. et SUHAdOLNIK, Natasa Vampelj (dir.), The Yields of Transition : Literature, Art and Philosophy in Early Medieval China. Newcastle Upon Tyne, Cambridge Scholars Publishing : 281292.

VuILLEUMIER, Victor (2011b). « Discours de 1'esprit, corps et culture dans la Chine moderne ». Études asiatiques/Asiatische Studien, vol. LXV, ${ }^{\circ} 1$ : 265-286.

VUILLEUMIER, Victor (2010). L'Écriture du corps déchiré dans la littérature chinoise moderne (1917-1949), thèse $n^{\circ}$ 695, Faculté des Lettres, Université de Genève.

VuILleuMIER, Victor (2006). «Intertextual Comparison between Lu Xun and Tao Yuanming : A Rewriting of Classical Poetry in Modern Chinese Prose Poetry ». In Issues of Far Eastern Literatures. Book of Papers of 2nd International Scientific Conference. Saint-Pétersbourg, Saint Petersburg State University Publishing House, t. 1 : 181-196.

WANG, David Der-wei (2004). The Monster That is History. History, Violence, and Fictional Writing in Twentieth-Century China. Berkeley, Los Angeles, Londres.

WANG, Dewei 王德威 (2003). Xiandai Zhongguo xiaoshuo shi jiang 現代中國小說十 講. Shanghai, Fudan daxue chubanshe. 
WANG, Ban (1997). The Sublime Figure of History. Aesthetics anf Politics in TwentiethCentury China. Stanford, Stanford University Press.

WANG, Hui 汪暉 (2008). Fankang juewang : Lu Xun ji qi wenxue shijie : zengdingban 反抗絕望：魯迅及其文學世界：增訂版. Pékin, Sanlian shudian.

WU, Yu 吴虞 (1982). 《Chiren yu lijiao»吃人與禮教 [1918]. In LI, Zongying et ZHANG, Mengyang (dir.), Liushinian lai Lu Xun yanjiu lunwenxuan 六十年來魯 迅研究論文選. Pékin, Zhongguo shehui kexue chubanshe, t. 1 : 2-6.

XIAO, Hong et GoldBlatT, Howard (trad.) (1979). The Field of Life and Death and Tales of Hulan River. Bloomington, Indiana University Press.

YUAN, Xingpei 袁行需, MENG, Erdong 孟二冬 et DINGFANG 丁放 (dir.) (1996). Zhongguo shixue tonglun 中國詩學通論. Hefei, Anhui jiaoyu chubanshe.

ZHANG, Xinying 張新穎 (2001). 20 shiji shangbanqi Zhongguo wenxuede xiandai yishi 20 世紀上半期中國文學的現代意識. Pékin, Sanlian.

ZHANG, Yinde (1992). Le Roman chinois moderne. Paris, PUF.

ZHUANGZI 莊子 et CHEN, Guying 陳鼓應 (éd.) (1998). Zhuangzi jin zhu jin yi 莊子今 注今譯. Pékin, Zhonghua shuju, 3 t.

\section{GLOSSAIRE}

A Q zhengzhuan 阿Q正傳

baguaquan 八卦拳

baihua 白話

baihuade wenzhang 白話的文章

benneng 本能

benwei 本味

$b i j i$ 筆記

biaoxian 表現

Binghou zatan 病後雜談

bopi 剥皮

butian 補天

cankui 慚愧

chaoren 超人

chensiren de xue he rou 陳死人的血和肉

Chu guan 出關

Cong huxu shuodao yachi 從鬊䰅說到牙齒

dai mutou 呆木頭

Doufu Xi Shi 豆腐西施

$d u$ 毒

duhai 毒害

duoshu 多數 
Ershisi xiaotu 二十四孝圖

feizao 肥白

$f u g u$ 復古

fuchou 復仇

fuqinde bing 父親的病

gaihui 改悔

Gao lao fuzi 高老夫子

Gemingjun 革命軍

gexing 個性

gongxing 宮刑

gudaide sirende hua 古代的死人的話

guwen 古文

Guxiang 故鄉

gui 鬼

guoke 過客

guominxing 國民性

Guo Moruo 郭沫若

guoshu 國術

guoxue 國學

guoyu 國語

hei'an 黑暗

$\mathrm{Hu}$ Shi 胡適

Huaijiu 懷舊

hun 魂

huoguolai 活過來

huorende chunshe zuowei yuanquan 活人的唇舌作為源泉

$j i k u$ 疾苦

jiangyao yongbao jiangyao shalu 將要擁抱將要殺翏

jiepou 解剖

jinrou 筋肉

jingshen 精神

jingshenjie zhi zhanshi 精神界之戰士

Junguomin yundong 軍國民運動

kaikou 開 $\square$

kanpo 看破

kexue 科學

kongxu 空虛

Kong Yiji 孔乙己

Kongzi 孔子

kouchun bu dong 口唇不動 
Victor Vuilleumier

kumen 苦悶

kumende xiangzheng 苦悶的象徵

Kuangren riji 狂人日記

Kuriyagawa Hakuson 廚川白村

lijiao 禮教

liren 立人

linghun 靈魂

lingjue 靈覺

$\mathrm{Lu} \mathrm{Ji}$ 陸機

mamude jingshen 麻木的精神

mianzi 面子

Mianzi he menqian 面子和門錢

minzuxing 民族性

Moluo shili shuo 魔羅詩力說

Mujiewen 墓碣文

nüexing 虐刑

Po e'sheng lun 破惡聲論

Qisi 起死

qiangzhuangde tige 強壯的體格

Qing 清

$q u$ jin $f u g u$ 取今復古

quke 軀殼

ren 人

Ren sheng xiangxiao 人生象學

renti 人體

ruzhe 儒者

shenjing 神經

sheng 生

sheng 聲

shengde kumen 生的苦悶

shengli 生理

shengming 生命

shengmingli 生命力

shengqi 生氣

shengsichang 生死場

$s h i$ 詩

shi yan zhi 詩言志

Shuo huxu 說鬞䰅

Shuo mianzi 說面子

Sibada xiaozhi 斯巴達小志 
Sibada zhi hun 斯巴達之魂

Sihou 死後

Sihuo 死火

$s u$ 俗

Takayama Chogyû 高山㯪牛

taohongse de pifu 桃紅色的皮膚

Tengye xiansheng 藤野先生

Tici 題辭

tizhi 體質

tiancai 天才

tianxia 天下

tipo meixue 體魄美學

Toufade gushi 頭髮的故事

Tongcheng 桐城

Tuibaixiande zhandong 頽敗線的顫動

Wei Jin 魏晉

weisheng 衛生

Weixin 維新

Wenhua pianzhi lun 文化偏至論

wenyan 文言

wenziyu 文字獄

Wushengde Zhongguo 無聲的中國

Wusi 五四

wuwu zhi zhen 無物之陣

$w u z h i$ 物質

Women xianzai zenyang zuo fuqin 我們現在怎樣做父親

Wo zhi jielie guan 我之節烈觀

xiati 下體

xiandaide huorende hua 現代的活人的話

xian wang zhi ze 先王之澤

Xiao Hong 蕭紅

Xiaojing 孝經

Xie zai Fen houmian 寫在墳後面

xin 心

xinli 心力

Xin wenhua yundong 新文化運動

xin wenxue 新文學

xinsheng 新生

xinsheng 心聲

xinsheng zhi zuo 新生之作 
Victor Vuilleumier

xinxue 心學

xinxue 新學

xin zong 新宗

Xu Zhimo 徐志摩

ya 雅

Yao 藥

yemande ren 野蠻的人

yeshi 野史

yijia yanjiu 醫家研究

$y i s h u$ 醫術

Yingde gaobie 影的告別

youbi 幽閉

youhun 遊魂

Yu Dafu 郁達夫

yuhen 餘痕

Yusi 語絲

yuyan 語言

Yuan qiang 原強

Zhang Taiyan 章太炎

Zheiyangde zhanshi 這樣的戰士

zhende ren 真的人

zhong wu xin gan 中無心肝

zhongzhi 眾治

Zhufu 祝福

Zixu 自序

Zhou Zuoren 周作人 